\title{
ON JARRATT'S FAMILY OF OPTIMAL FOURTH-ORDER ITERATIVE METHODS AND THEIR DYNAMICS
}

\author{
Changbum Chun,,$\ddagger$ Beny Neta ${ }^{\dagger, \S}$ and Sujin Kim*, \\ *Department of Mathematics \\ Sungkyunkwan University \\ Suwon 440-746, Republic of Korea \\ ${ }^{\dagger}$ Naval Postgraduate School \\ Department of Applied Mathematics \\ Monterey, CA 93943, USA \\ †cbchun@skku.edu \\ §bneta@nps.edu \\ ^sjylover012@hanmail.net
}

Received October 23, 2013

Accepted June 29, 2014

Published July 29, 2014

\begin{abstract}
P. Jarratt has developed a family of fourth-order optimal methods. He suggested two members of the family. The dynamics of one of those was discussed previously. Here we show that the family can be written using a weight function and analyze all members of the family to find the best performer.
\end{abstract}

Keywords: Iterative Methods; Order of Convergence; Weight Functions; Simple Roots.

\section{INTRODUCTION}

There is a vast literature on the solution of nonlinear equations and nonlinear systems, see for example Ostrowski, ${ }^{1}$ Traub, ${ }^{2} \mathrm{Neta}^{3}$ and the recent book by Petković et al. ${ }^{4}$ and references therein. Most of the algorithms are for finding a simple root

\footnotetext{
${ }^{\S}$ Corresponding author.
} 
of a nonlinear equation $f(x)=0$, i.e., for a root $\alpha$ we have $f(\alpha)=0$ and $f^{\prime}(\alpha) \neq 0$. Methods are generally compared by their efficiency index, defined by

$$
I=p^{1 / d},
$$

where $p$ is the order of convergence and $d$ is the number of function- (and derivative-) evaluation per step. For example, the well-known Newton's method given by

$$
x_{n+1}=x_{n}-u_{n},
$$

where

$$
u_{n}=\frac{f\left(x_{n}\right)}{f^{\prime}\left(x_{n}\right)},
$$

is of second order and requires the computation of $f$ and $f^{\prime}$ and, thus, its efficiency index is $I=\sqrt{2}=$ 1.4142 .

One way to improve the order of convergence is by including higher order derivatives. Unfortunately this will not increase the efficiency much unless we go to multistep methods. For example, Halley's $\operatorname{method}^{5}$ is of third order requiring the computation of $f$ and its first two derivatives. Therefore $I=3^{1 / 3}=1.442$ which is only slightly higher than that of Newton's method. On the other hand the two step method developed by Chun et $a .^{6}$ and given by:

$$
\begin{gathered}
y_{n}=x_{n}-\frac{2}{3} u_{n}, \\
x_{n+1}=x_{n}-q\left(t_{n}\right) u_{n},
\end{gathered}
$$

where $t_{n}=\frac{3}{2} \frac{f^{\prime}\left(x_{n}\right)-f^{\prime}\left(y_{n}\right)}{f^{\prime}\left(x_{n}\right)}$ does not require second derivatives.

There is flexibility in choosing the weight function $q(t)$, in fact, one can find several choices in the literature. It is of order 4 if

$$
q(0)=1, \quad q^{\prime}(0)=1 / 2, \quad q^{\prime \prime}(0)=1 .
$$

Therefore its efficiency is $I=4^{1 / 3}=1.587$ slightly higher than Halley's method. The error relation is given by

$$
\begin{aligned}
e_{n+1}= & {\left[\left(5-\frac{4}{3} q^{\prime \prime \prime}(0)\right) c_{2}^{3}-c_{2} c_{3}+\frac{1}{9} c_{4}\right] e_{n}^{4} } \\
& +O\left(e_{n}^{5}\right),
\end{aligned}
$$

where

$$
c_{i}=\frac{f^{(i)}(\alpha)}{i ! f^{\prime}(\alpha)} .
$$

Jarratt $^{7}$ has developed a family of optimal fourthorder methods given by

$$
\begin{aligned}
x_{n+1}= & x_{n}-a_{1} u_{n}-a_{2} \frac{f\left(x_{n}\right)}{f^{\prime}\left(x_{n}-\frac{2}{3} u_{n}\right)} \\
& -\frac{f\left(x_{n}\right)}{b_{1} f^{\prime}\left(x_{n}\right)+b_{2} f^{\prime}\left(x_{n}-\frac{2}{3} u_{n}\right)},
\end{aligned}
$$

where the parameters $a_{1}, a_{2}, b_{1}$ and $b_{2}$ satisfy the following

$$
\begin{aligned}
& a_{1}=\frac{1}{4}\left(1+\frac{3}{2 \theta}\right) \\
& a_{2}=\frac{3}{4}\left(1-\frac{1}{2(\theta-1)}\right) \\
& b_{1}=\frac{b_{2}}{\theta}-b_{2} \\
& b_{2}=\frac{8 \theta^{2}}{3}(\theta-1) .
\end{aligned}
$$

For $\theta=0$ and $\theta=1$ the family is only third order.

It is easy to show that this family can be written as (4) with the weight function $q(t)$ given by

$$
q\left(t_{n}\right)=\frac{1+d t_{n}+e t_{n}^{2}}{1+b t_{n}+c t_{n}^{2}}
$$

where $t_{n}=\frac{3}{2} \frac{f^{\prime}\left(x_{n}\right)-f^{\prime}\left(y_{n}\right)}{f^{\prime}\left(x_{n}\right)}$ and $y_{n}$ is given by the first step of (4). For example, for the case that $d=0$, $b=-0.5, c=-0.25$ and $e=0$ we have the method (24) in. ${ }^{6}$ For Jarratt's optimal fourth-order family $(\theta \neq 0, \theta \neq 1)$ we have

$$
\begin{aligned}
& d=-\frac{2}{3} \theta-\frac{1}{6} \\
& e=\frac{1}{9} \theta+\frac{1}{6} \\
& b=-\frac{2}{3}(\theta+1) \\
& c=\frac{4}{9} \theta .
\end{aligned}
$$

In this paper, we would like to look at a more general family (8) satisfying (5), i.e.

$$
q(t)=\frac{1+\left(2 e-2 c-\frac{1}{2}\right) t+e t^{2}}{1+(2 e-2 c-1) t+c t^{2}} .
$$

Clearly, for Jarratt's family the parameters $c$ and $e$ are interdependent and

$$
c=4 e-\frac{2}{3} .
$$


In the next two sections, we analyze the basin of attraction of our fourth order family of methods to find out what is the best choice for $c$ and $e$. The idea of using basins of attraction was initiated by Stewart $^{8}$ and followed by the works of Amat et al. ${ }^{9-12}$ Scott et al. ${ }^{13}$ Chun et al. ${ }^{6}$ Chicharro et al. ${ }^{14}$ Cordero et al. ${ }^{15}$ and Neta et al. ${ }^{16}$ The only papers comparing basins of attraction for methods to obtain multiple roots is due to Neta et al., ${ }^{17}$ and Neta and Chun. ${ }^{18}$

We will choose the weight function to restrict the extraneous fixed points to the imaginary axis. This is a result of analyzing King's family of methods. ${ }^{16}$

\section{CORRESPONDING CONJUGACY MAPS FOR QUADRATIC POLYNOMIALS}

Given two maps $f$ and $g$ from the Riemann sphere into itself, an analytic conjugacy between the two maps is a diffeomorphism $h$ from the Riemann sphere onto itself such that $h \circ f=g \circ h$. Here we consider only quadratic polynomials.

Theorem 1. For a rational map $R_{p}(z)$ arising from method (4) with $q$ given by (9) applied to $p(z)=(z-a)(z-b), a \neq b, R_{p}(z)$ is conjugate via the Möbius transformation given by $M(z)=\frac{z-a}{z-b}$ to

$$
S(z)=\frac{-z^{2}+(4 c-4 e-2) z-1-8 e+4 c}{(-1-8 e+4 c) z^{2}+(4 c-4 e-2) z-1} z^{4} .
$$

Proof. Let $p(z)=(z-a)(z-b), a \neq b$ and let $M$ be the Möbius transformation given by $M(z)=$ $\frac{z-a}{z-b}$ with its inverse $M^{-1}(u)=\frac{u b-a}{u-1}$, which may be considered as a map from $\mathbb{C} \cup\{\infty\}$. We then have

$$
\begin{aligned}
S(u) & =M \circ R_{p} \circ M^{-1}(u)=M \circ R_{p}\left(\frac{u b-a}{u-1}\right) \\
& =\frac{-u^{2}+(4 c-4 e-2) u-1-8 e+4 c}{(-1-8 e+4 c) u^{2}+(4 c-4 e-2) u-1} u^{4} .
\end{aligned}
$$

The question now is: Can we find parameters such that the conjugacy map is a monomial? As a special case of Theorem 1 we see that

(1) $S(u)=u^{6}$ when $c=0.75, e=0.25$;

(2) $S(u)=u^{5}$ when $c=0.25, e=0$;

(3) $S(u)=-u^{5}$ when $c=1.25, e=0.5$;
(4) $S(u)=u^{4}$ when $c=2 e, e=e$; and

(5) $S(u)=-u^{4}$ when $c=0.5, e=0$.

The case for which $c=2 e$ leads to $S(u)=u^{4}$. If $c=2 e, e=1 / 3$ we have Jarratt's method with $\theta=3 / 2$. The case for which $c=e=2 / 9$ leads to the well-known Jarratt's fourth-order method (with $\theta=1 / 2)^{7}$

$$
\begin{aligned}
y_{n} & =x_{n}-\frac{2}{3} u_{n}, \\
x_{n+1} & =x_{n}-\left[1-\frac{3}{2} \frac{f^{\prime}\left(y_{n}\right)-f^{\prime}\left(x_{n}\right)}{3 f^{\prime}\left(y_{n}\right)-f^{\prime}\left(x_{n}\right)}\right] u_{n} .
\end{aligned}
$$

In this case $S(u)$ is not a polynomial. The case for which $c=-0.25$ and $e=0$ gives the method (24) proposed in Ref. 6.

\section{EXTRANEOUS FIXED POINTS}

As mentioned earlier, in solving a nonlinear equation iteratively we are looking for fixed points which are zeros of the given nonlinear function. Many multipoint iterative methods have fixed points that are not zeros of the function of interest. Thus, it is imperative to investigate the number of extraneous fixed points, their location and their properties. In the method described in this paper, the parameters $c$ and $e$ can be chosen to position the extraneous fixed points on the imaginary axis.

The fourth order methods discussed here can be written as

$$
x_{n+1}=x_{n}-\frac{f\left(x_{n}\right)}{f^{\prime}\left(x_{n}\right)} H_{f}\left(x_{n}\right),
$$

where

$$
H_{f}\left(x_{n}\right)=q\left(t_{n}\right)=q\left(t\left(x_{n}\right)\right) .
$$

Clearly the root $\alpha$ of $f(x)$ is a fixed point of the method. The points $\xi \neq \alpha$ at which $H_{f}(\xi)=0$ are also fixed points of the family.

We have tried several possibilities for the function $q$ and have computed the extraneous fixed points. One would like to have the extraneous fixed points on the imaginary axis which is the boundary between the two roots of the quadratic polynomial.

When $q$ given by (9), $H_{f}$ is given by

$$
H_{f}(z)=\frac{(4 c-5 e-3) z^{4}+(-4 c+6 e-1) z^{2}-e}{(3 c-2-4 e) z^{4}+(-2 c-2+4 e) z^{2}-c} .
$$

The extraneous fixed points are functions of $c$ and $e$. We have searched values of the parameters so 
Table 1 The Four Extraneous Fixed Points for Selected Values of $c$ and $e$.

\begin{tabular}{cccccc}
\hline Index & $\boldsymbol{c}$ & $\boldsymbol{e}$ & Roots 1, 2 & Roots 3, 4 & \\
\hline 1 & -0.25 & 0 & 0,0 & 0,0 & $(24)$ \\
2 & 0 & 0 & $\pm 0.5773503 i$ & & $u^{4}$ \\
3 & 0.25 & 0 & $\pm i$ & 0,0 & $u^{5}$ \\
4 & 0.5 & 0 & $\pm 1.7320508 i$ & 0,0 & $-u^{4}$ \\
5 & $2 / 3$ & $1 / 3$ & $\pm 0.5773503 i$ & & $u^{4}$ \\
6 & 0.75 & 0.25 & $\pm 1.3763819 i$ & $\pm 0.3249197 i$ & $u^{6}$ \\
7 & 0.76 & 0.01 & $\pm 19.9498744 i$ & $\pm 0.0501256 i$ & Largest \\
8 & 0.76 & 0.38 & $\pm 0.7828814 i$ & $\pm 0.5773503 i$ & $c=2 e, u^{4}$ \\
9 & 1.25 & 0.5 & $\pm 2.4142136 i$ & $\pm 0.4142136 i$ & $-u^{5}$ \\
10 & 1.26 & 0.41 & $\pm 18.9178603 i$ & $\pm 0.3384698 i$ & Largest \\
11 & 1.82 & 0.91 & $\pm 0.5773503 i$ & & $c=2 e, u^{4}$ \\
12 & $2 / 9$ & $2 / 9$ & & & $(12)$ \\
\hline
\end{tabular}

that the extraneous fixed points are on the imaginary axis. We found that $-0.25 \leqslant c \leqslant 5.75$ and $0 \leqslant e \leqslant 4$. In Table 1 we give a list of the extraneous fixed points for selected values of the parameters $c$ and $e$. All these points lie on the imaginary axis except the last case where $c=e=2 / 9$ corresponding to (12). The first one corresponds to (24) of Ref. 6. The second, third, fourth, fifth, sixth and ninth are those that give a monomial map. The seventh is a case where the extraneous fixed points are farthest from the origin. The tenth case is for which the sum of the magnitudes of the extraneous fixed points is the largest. We took two other cases (eighth and eleventh) with $c=2 e$, for which also have a monomial map.

All these fixed points are repulsive.

In the next section we plot the basins of attraction for these cases to find the best performer.

\section{NUMERICAL EXPERIMENTS}

We have used the 12 members of the family of methods for six different polynomials.

Example 1. In our first example, we have taken the polynomial to be

$$
p_{1}(z)=z^{2}-1
$$

whose roots $z= \pm 1$ are both real. The results are presented in Figs. 1-12. Figure 1 has large black regions indicating the method does not converge in 40 iterations starting at those points. Two other schemes have black regions but not as large. These are the methods with $c=0.76$ and $e=0.01$ (Fig. 7) and the one with $c=1.26$ and $e=0.41$ (Fig. 10). The best scheme is the one with $c=$

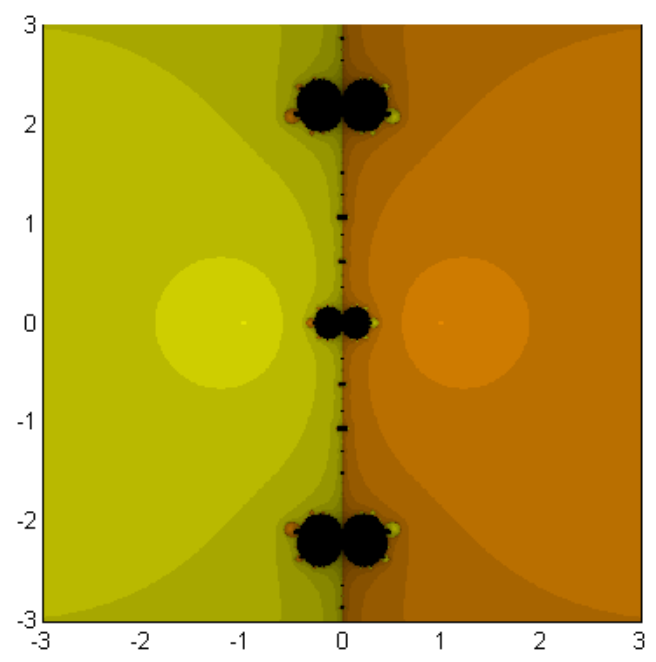

Fig. 1 Our method with $c=-0.25$ and $e=0$ for the roots of the polynomial $z^{2}-1$.

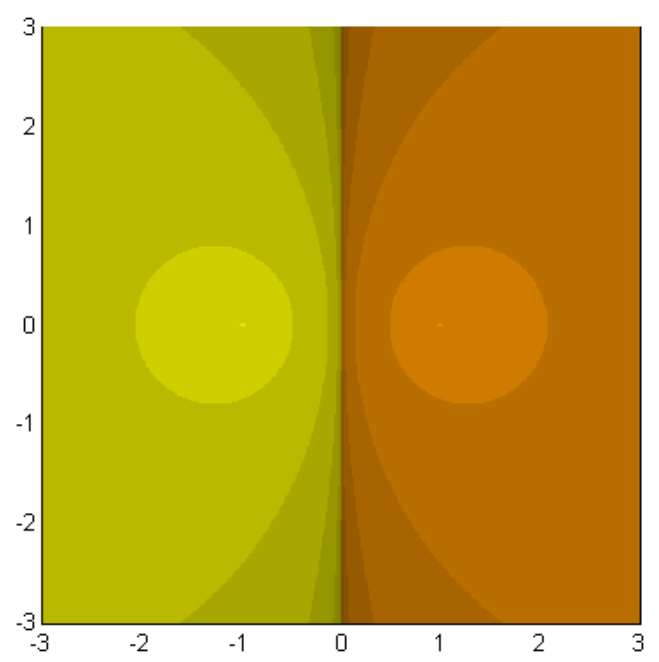

Fig. 2 Our method with $c=0$ and $e=0$ for the roots of the polynomial $z^{2}-1$.

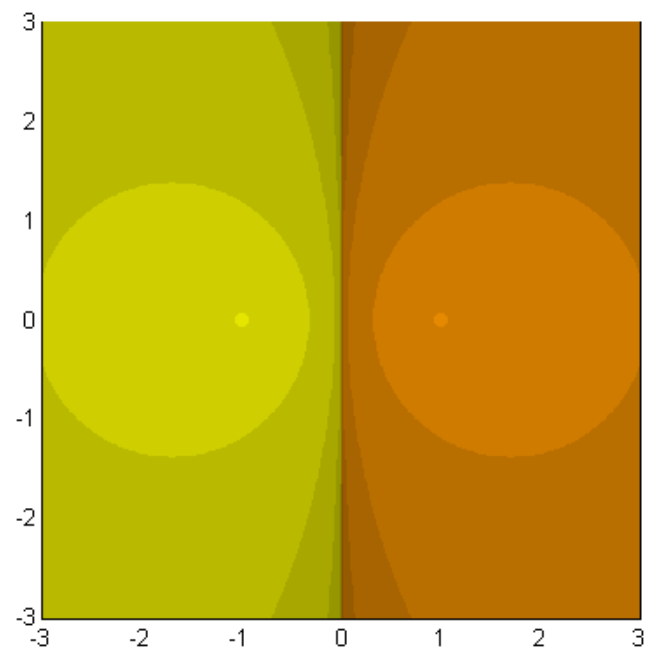

Fig. 3 Our method with $c=0.25$ and $e=0$ for the roots of the polynomial $z^{2}-1$. 


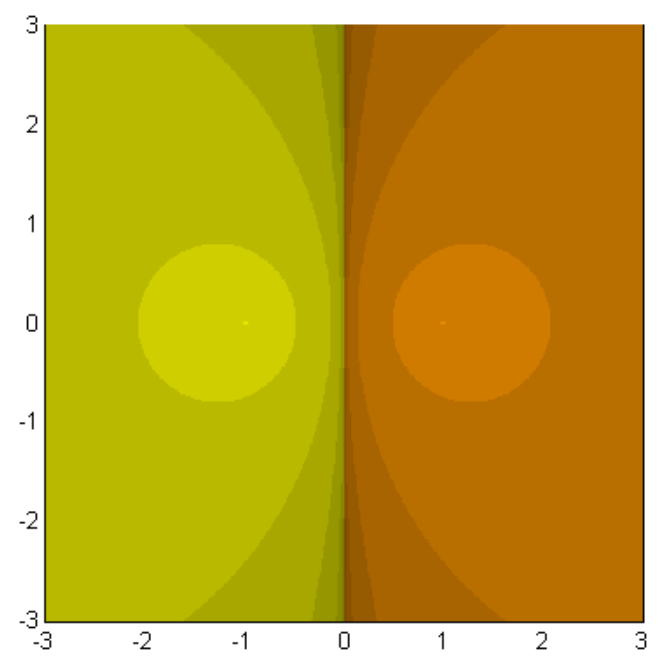

Fig. 4 Our method with $c=0.5$ and $e=0$ for the roots of the polynomial $z^{2}-1$.

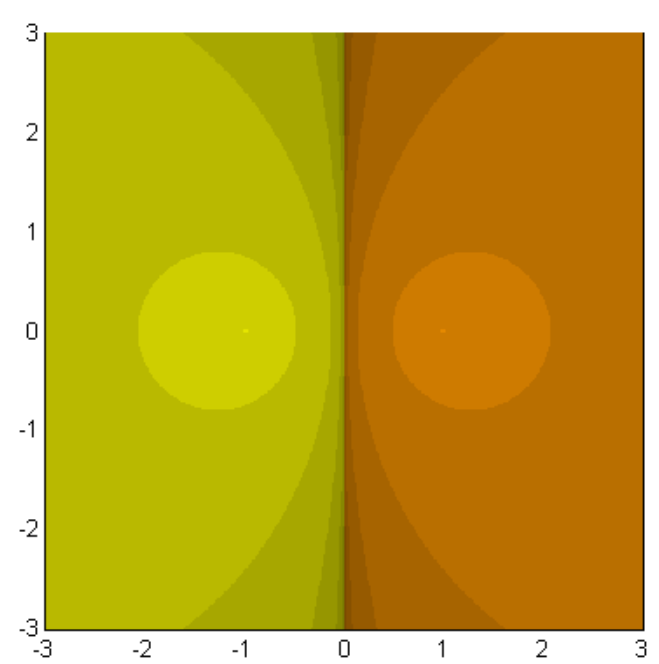

Fig. 5 Jarratt's method with $c=2 / 3$ and $e=1 / 3$ for the roots of the polynomial $z^{2}-1$.

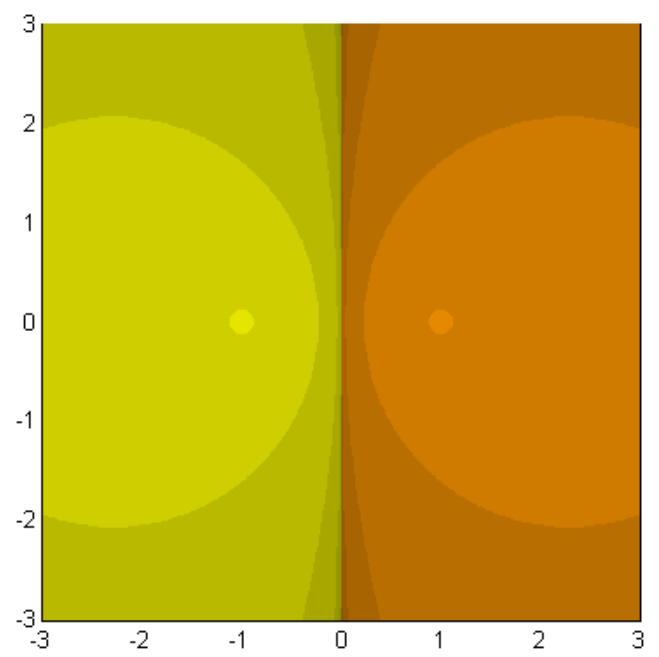

Fig. 6 Our method with $c=0.75$ and $e=0.25$ for the roots of the polynomial $z^{2}-1$.

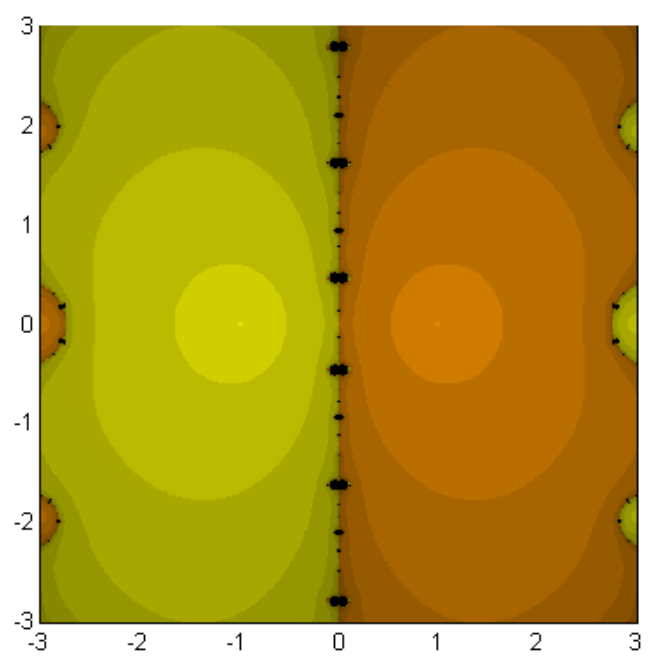

Fig. 7 Our method with $c=0.76$ and $e=0.01$ for the roots of the polynomial $z^{2}-1$.

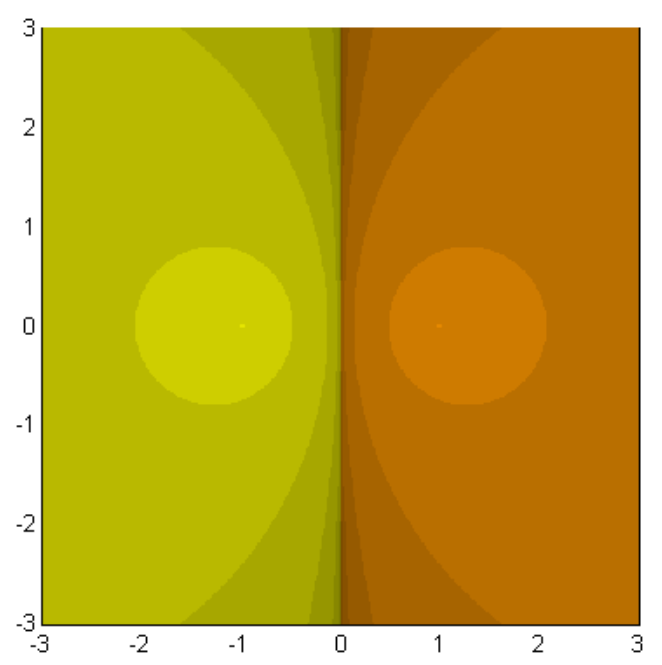

Fig. 8 Our method with $c=0.76$ and $e=0.38$ for the roots of the polynomial $z^{2}-1$.

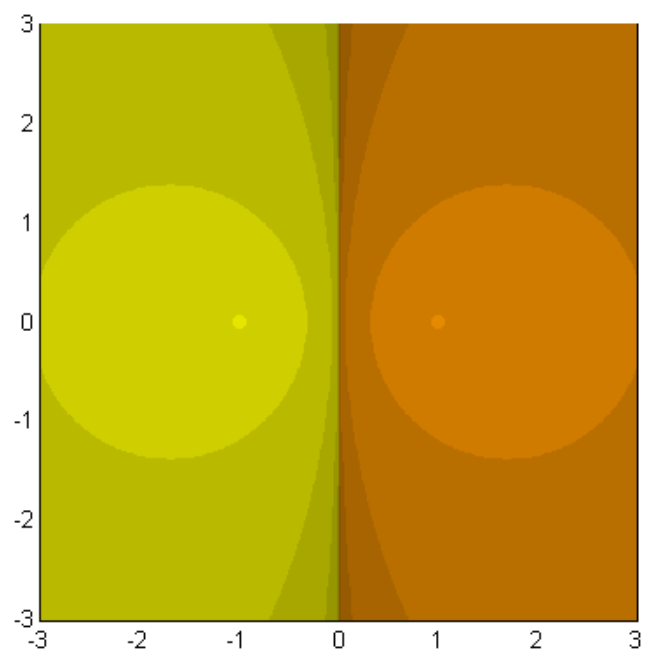

Fig. 9 Our method with $c=1.25$ and $e=0.5$ for the roots of the polynomial $z^{2}-1$. 


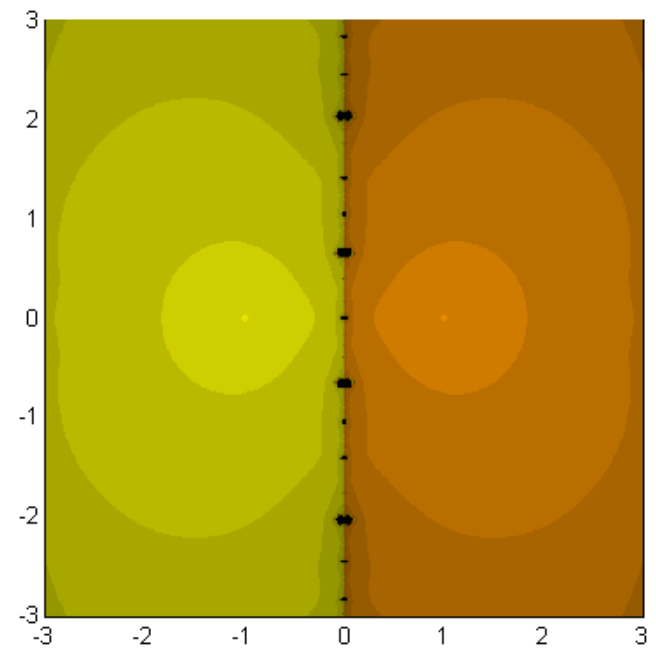

Fig. 10 Our method with $c=1.26$ and $e=0.41$ for the roots of the polynomial $z^{2}-1$.

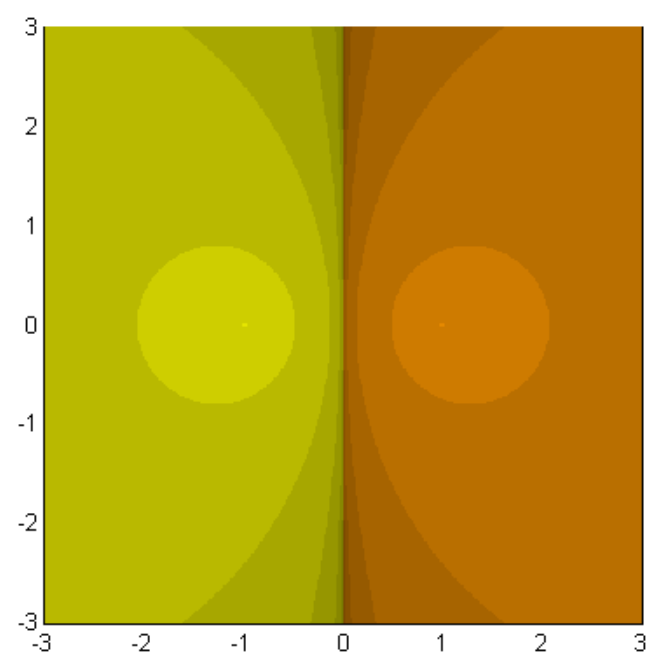

Fig. 11 Our method with $c=1.82$ and $e=0.91$ for the roots of the polynomial $z^{2}-1$.

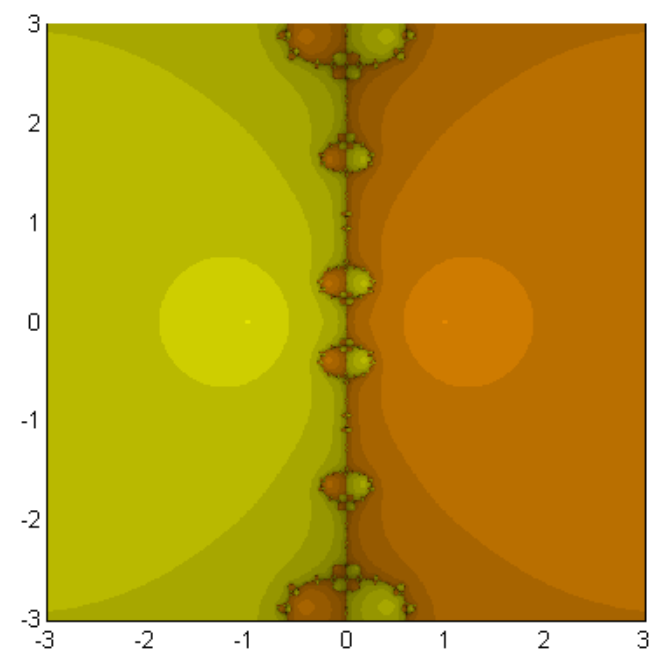

Fig. 12 Jarratt's method with $c=2 / 9$ and $e=2 / 9$ for the roots of the polynomial $z^{2}-1$.
$0.75, e=0.25$ (Fig. 6) which has a monomial conjugacy map $u^{6}$. The next group includes the scheme with $c=0.25, e=0$ (Fig. 3) and $c=1.25, e=0.5$ (Fig. 9) having a conjugacy map $\pm u^{5}$. The third group of schemes includes the method with $c=$ $e=0$ (Fig. 2), with $c=0.5, e=0$ (Fig. 4), with $c=2 / 3, e=1 / 3$ (Fig. 5), with $c=0.76, e=0.38$ (Fig. 8) and with $c=1.82, e=0.91$ (Fig. 11). These are methods which have a monomial conjugacy map $\pm u^{4}$.

Figure 12 is for Jarratt's method for which the extraneous fixed points are not on the imaginary axis. It can be seen that there are starting points on the right half that converge to the left and vice versa. There are no black regions in this example, but also the results are not as good as say in the other Jarratt's method (Fig. 5).

Example 2. In the second example we have taken a cubic polynomial with the three roots of unity, i.e.

$$
p_{2}(z)=z^{3}-1 \text {. }
$$

The results are presented in Figs. 13-24. The worst methods are the fourth (Fig. 16) and the ninth (Fig. 21). The first method (Fig. 13) and the seventh (Fig. 19) are only slightly better. The best are the second (Fig. 14), the fifth (Fig. 17), the eighth (Fig. 20) and the eleventh (Fig. 23), satisfying $c=2 e$. The next best are the sixth (Fig. 18) and the twelfth (Fig. 24) followed by the tenth (Fig. 22) and the third (Fig. 15).

Example 3. In the third example we have taken a polynomial of degree 4 with 4 real roots at $\pm 1, \pm i$,

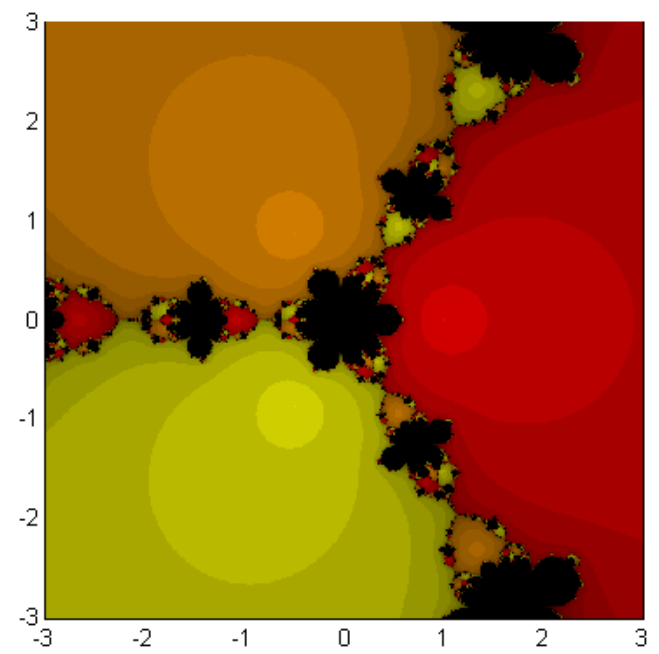

Fig. 13 Our method with $c=-0.25$ and $e=0$ for the roots of the polynomial $z^{3}-1$. 


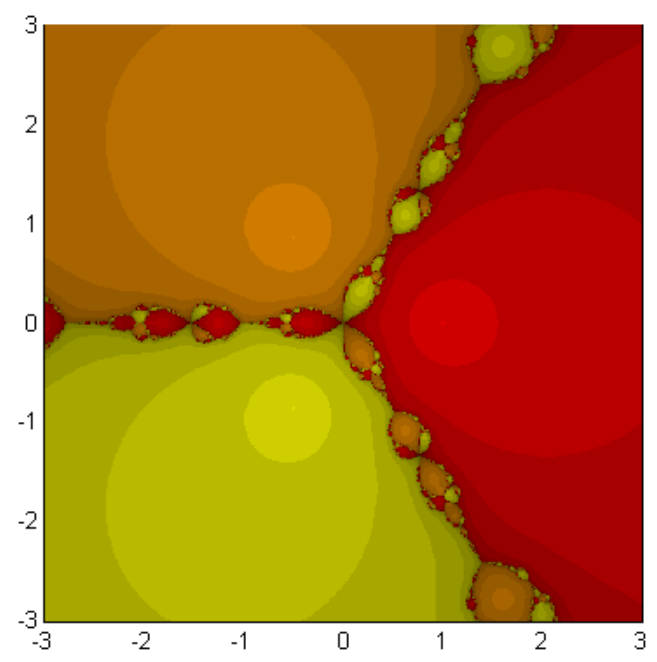

Fig. 14 Our method with $c=0$ and $e=0$ for the roots of the polynomial $z^{3}-1$.

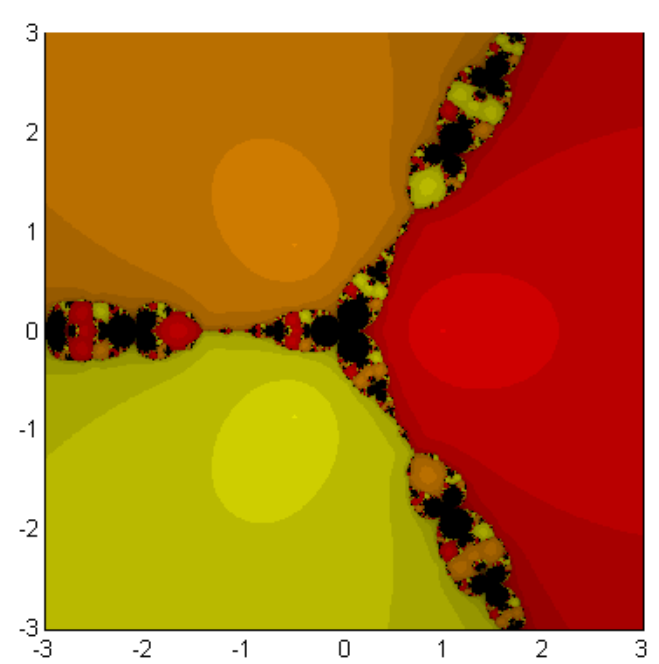

Fig. 15 Our method with $c=0.25$ and $e=0$ for the roots of the polynomial $z^{3}-1$.

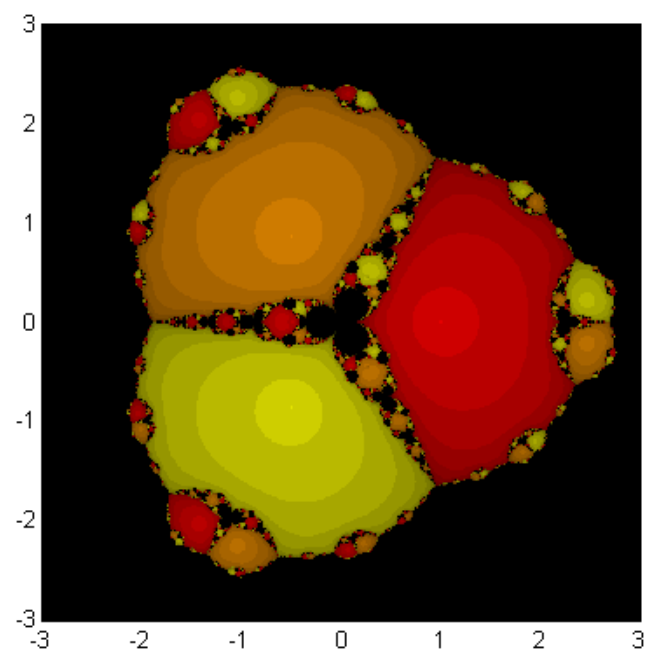

Fig. 16 Our method with $c=0.5$ and $e=0$ for the roots of the polynomial $z^{3}-1$.

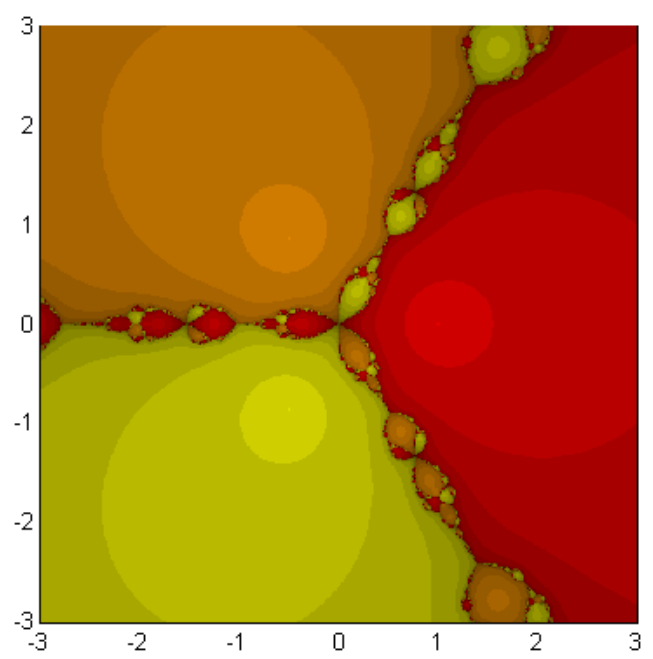

Fig. 17 Jarratt's method with $c=2 / 3$ and $e=1 / 3$ for the roots of the polynomial $z^{3}-1$.

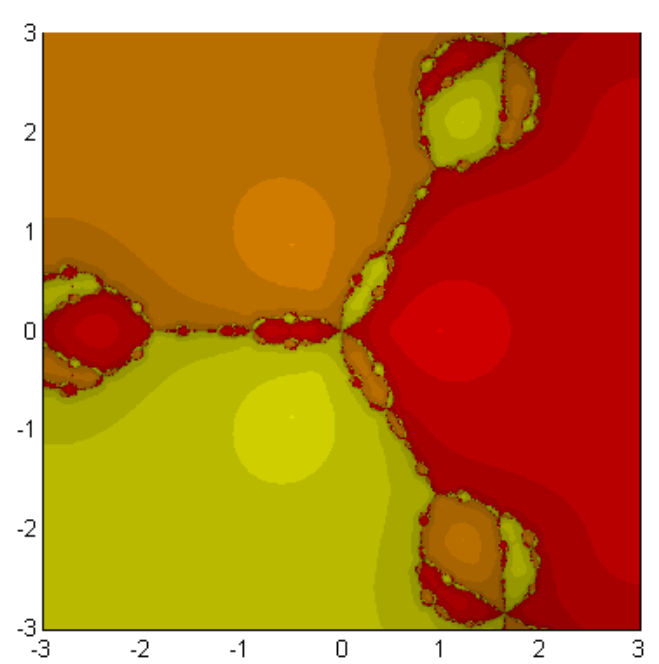

Fig. 18 Our method with $c=0.75$ and $e=0.25$ for the roots of the polynomial $z^{3}-1$.

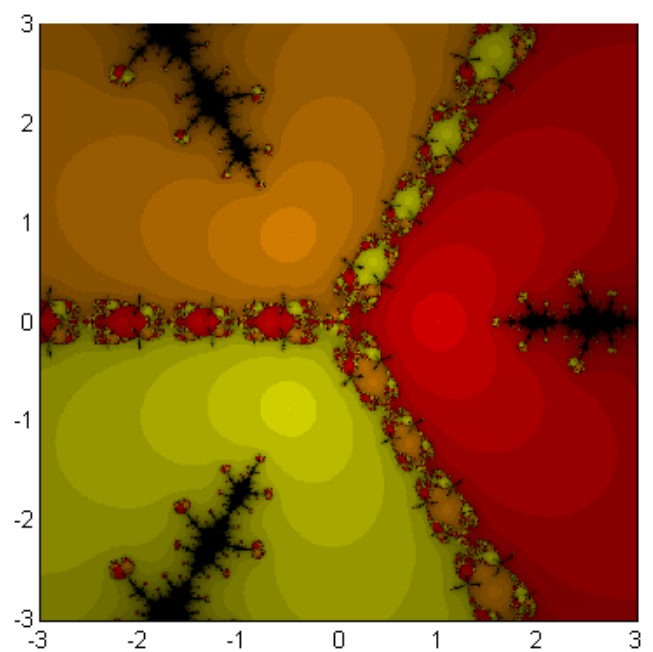

Fig. 19 Our method with $c=0.76$ and $e=0.01$ for the roots of the polynomial $z^{3}-1$. 


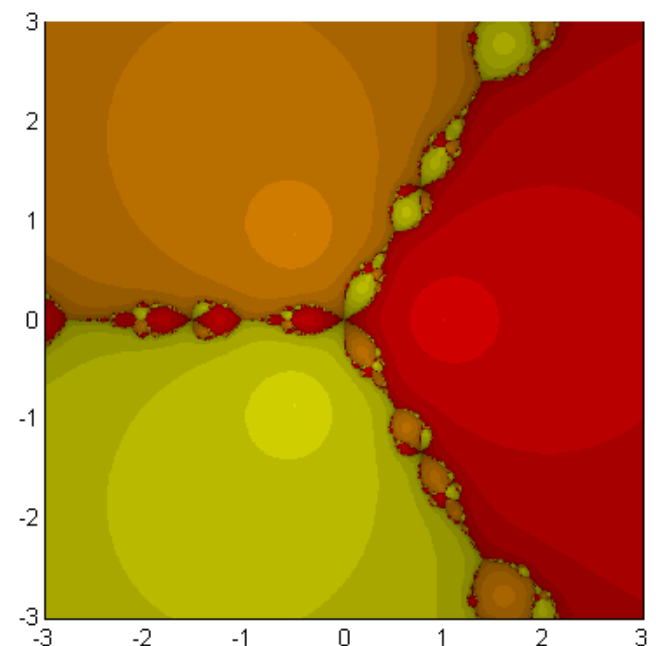

Fig. 20 Our method with $c=0.76$ and $e=0.38$ for the roots of the polynomial $z^{3}-1$.

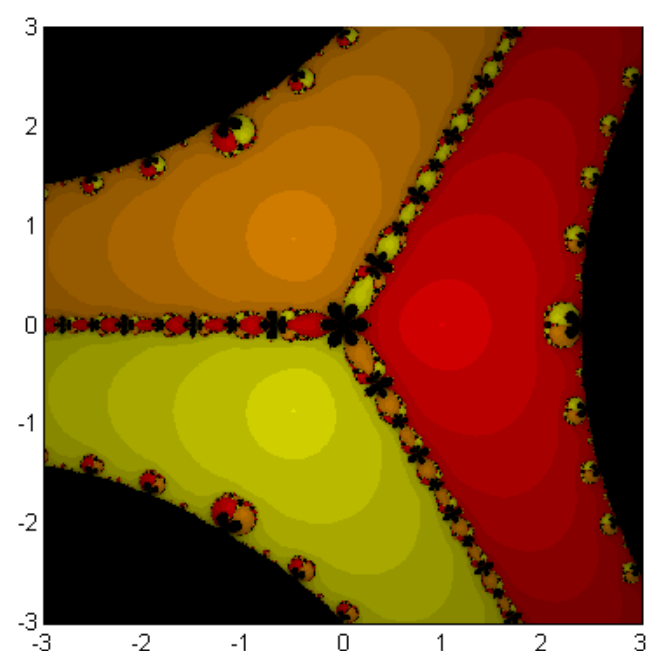

Fig. 21 Our method with $c=1.25$ and $e=0.5$ for the roots of the polynomial $z^{3}-1$.

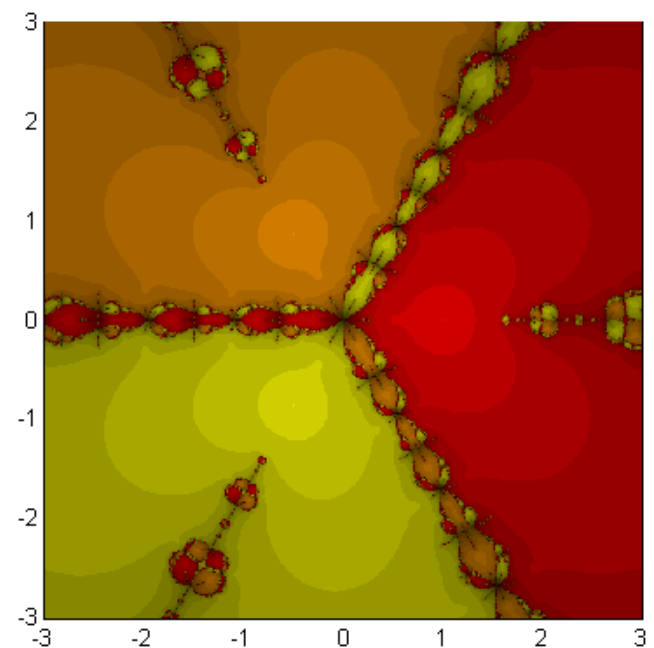

Fig. 22 Our method with $c=1.26$ and $e=0.41$ for the roots of the polynomial $z^{3}-1$.

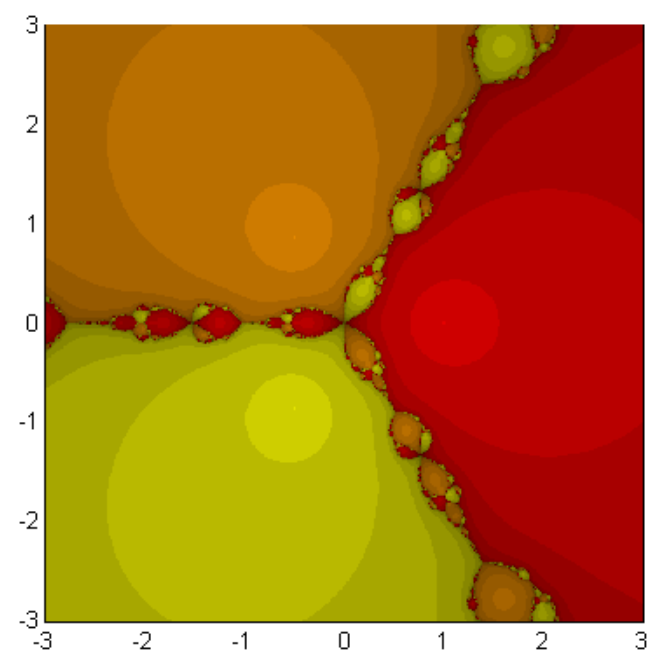

Fig. 23 Our method with $c=1.82$ and $e=0.91$ for the roots of the polynomial $z^{3}-1$.

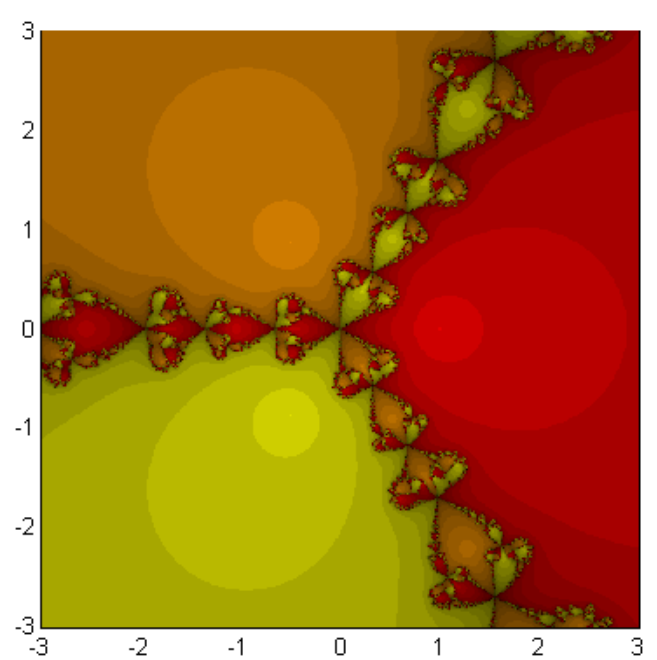

Fig. 24 Jarratt's method with $c=2 / 9$ and $e=2 / 9$ for the roots of the polynomial $z^{2}-1$.

i.e.

$$
p_{3}(z)=z^{4}-1 \text {. }
$$

The results are presented in Figs. 25-36. The worst methods are the first (Fig. 25), the fourth (Fig. 28) and the ninth (Fig. 33). The third method (Fig. 27) is only slightly better. The best ones are the second (Fig. 26), the fifth (Fig. 29), the eighth (Fig. 32) and the eleventh (Fig. 35). The next best are the tenth (Fig. 34) and the twelfth (Fig. 36), followed by the seventh (Fig. 31) and the sixth (Fig. 30).

At this point we will remove the four worst schemes and continue with the others. We remove the method with $c=-0.25, e=0$ since it was the worst or second worst in all three examples. We remove the one with $c=1.25, e=0.5$ because it was the worst in two examples and only mediocre 


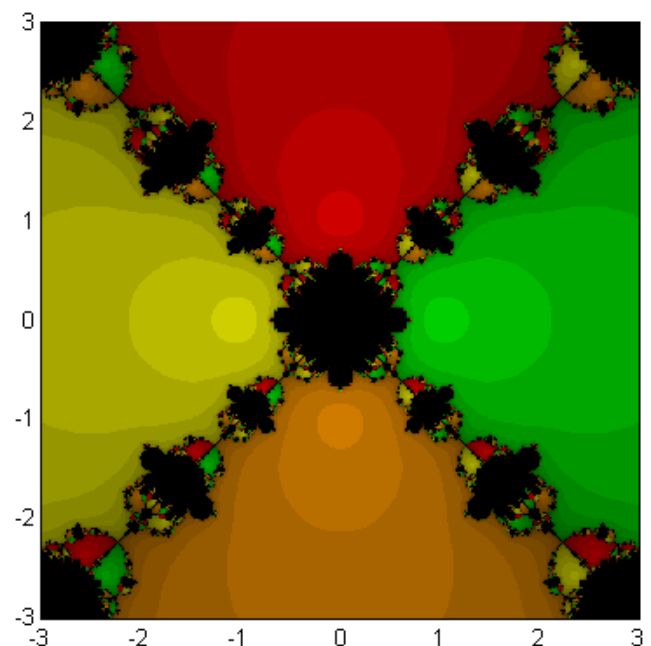

Fig. 25 Our method with $c=-0.25$ and $e=0$ for the roots of the polynomial $z^{4}-1$.

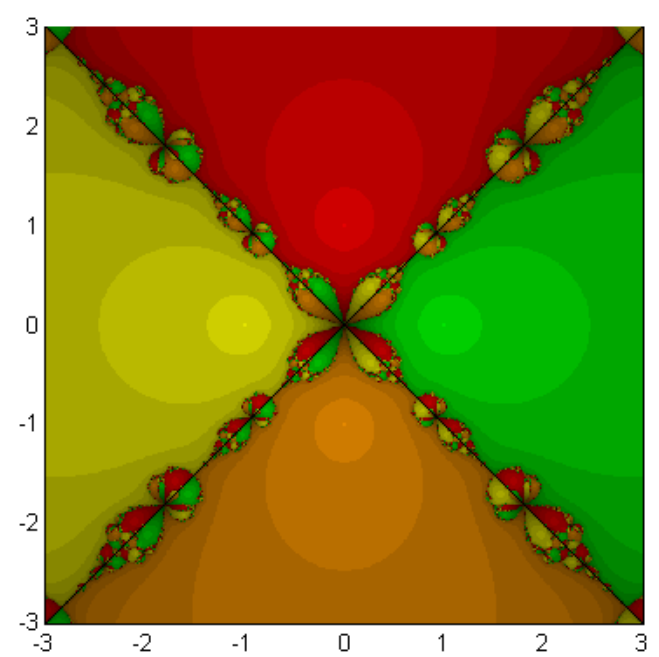

Fig. 26 Our method with $c=0$ and $e=0$ for the roots of the polynomial $z^{4}-1$.

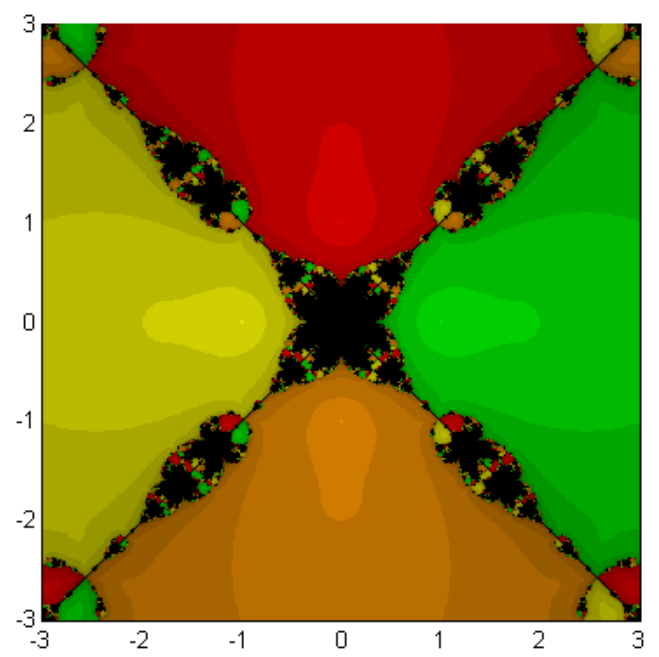

Fig. 27 Our method with $c=0.25$ and $e=0$ for the roots of the polynomial $z^{4}-1$.

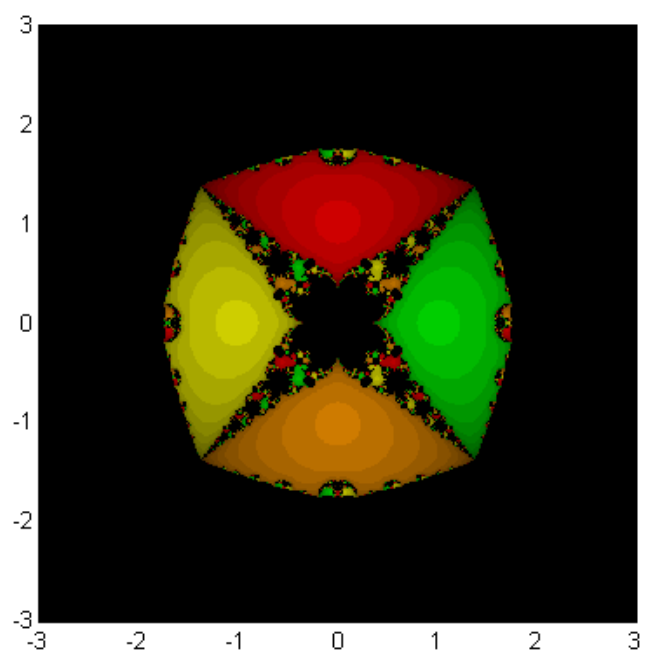

Fig. 28 Our method with $c=0.5$ and $e=0$ for the roots of the polynomial $z^{4}-1$.

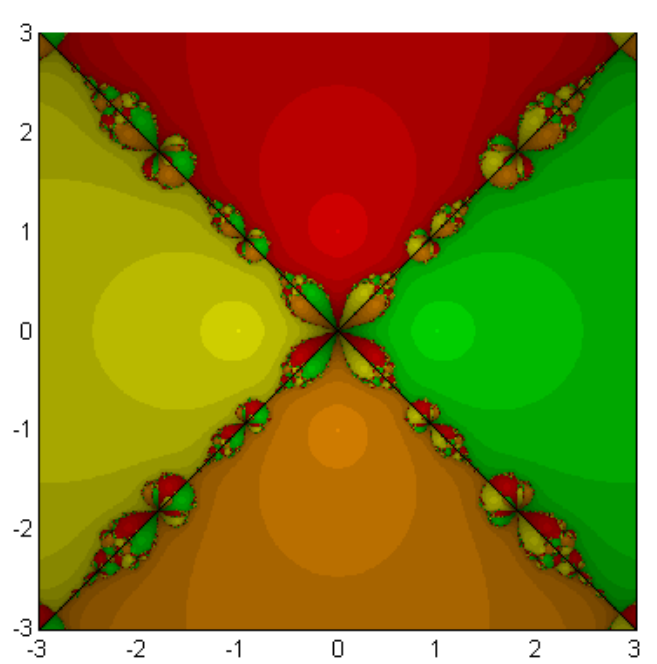

Fig. 29 Jarratt's method with $c=2 / 3$ and $e=1 / 3$ for the roots of the polynomial $z^{4}-1$.

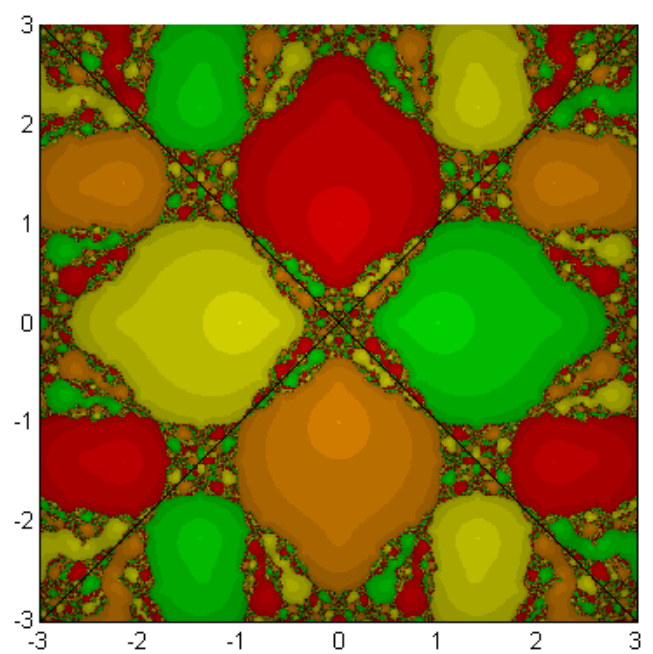

Fig. 30 Our method with $c=0.75$ and $e=0.25$ for the roots of the polynomial $z^{4}-1$. 
C. Chun, B. Neta \&S S. Kim

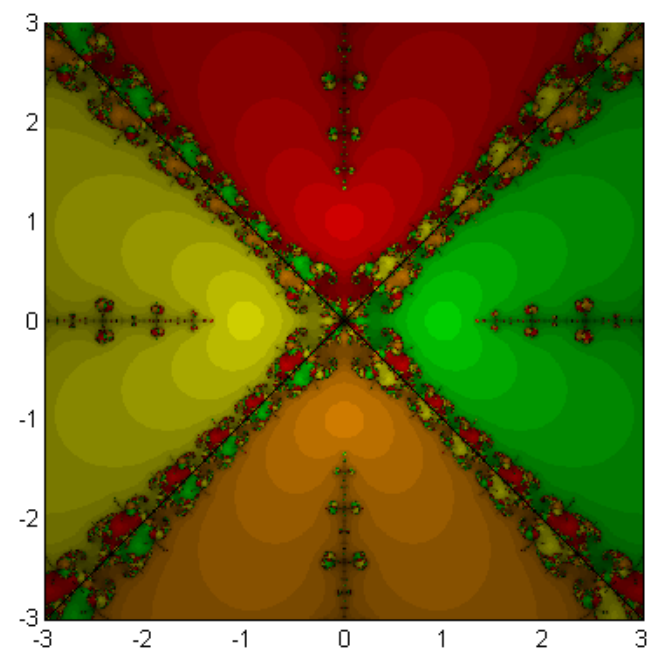

Fig. 31 Our method with $c=0.76$ and $e=0.01$ for the roots of the polynomial $z^{4}-1$.

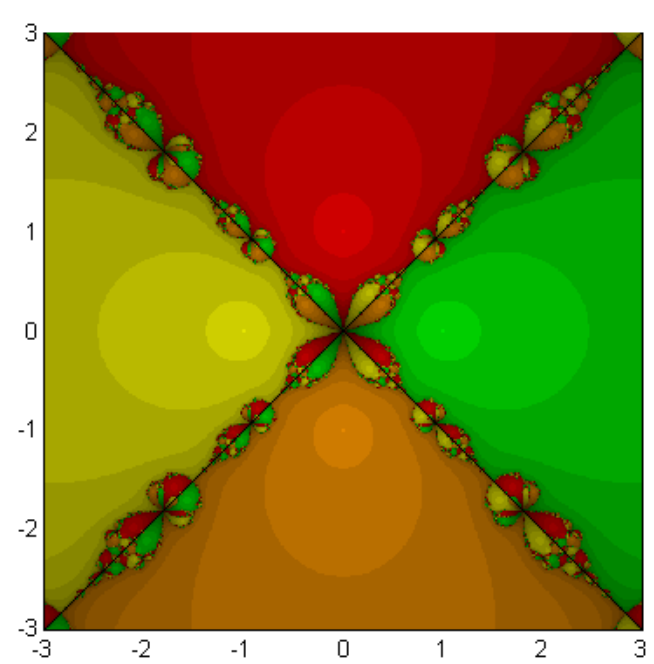

Fig. 32 Our method with $c=0.76$ and $e=0.38$ for the roots of the polynomial $z^{4}-1$.

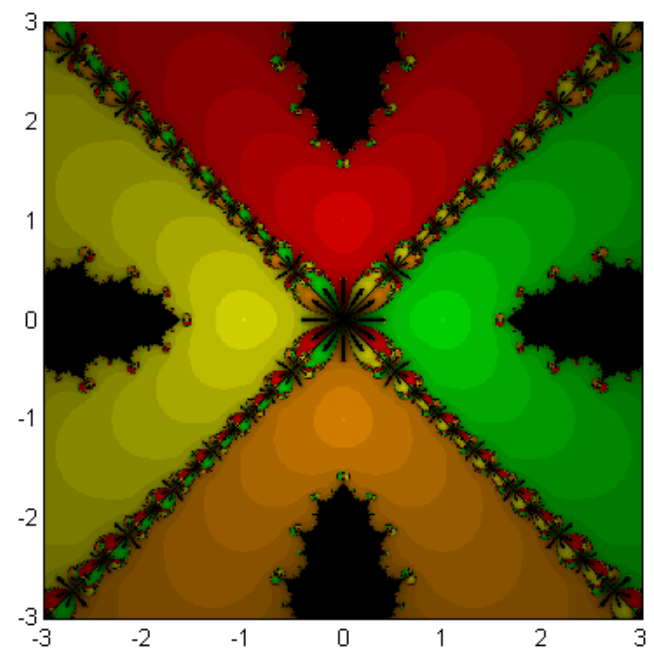

Fig. 33 Our method with $c=1.25$ and $e=0.5$ for the roots of the polynomial $z^{4}-1$.

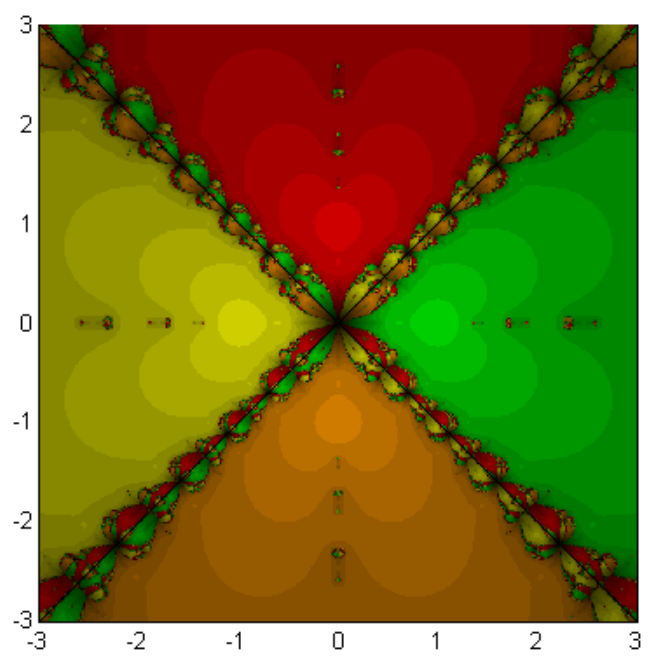

Fig. 34 Our method with $c=1.26$ and $e=0.41$ for the roots of the polynomial $z^{4}-1$.

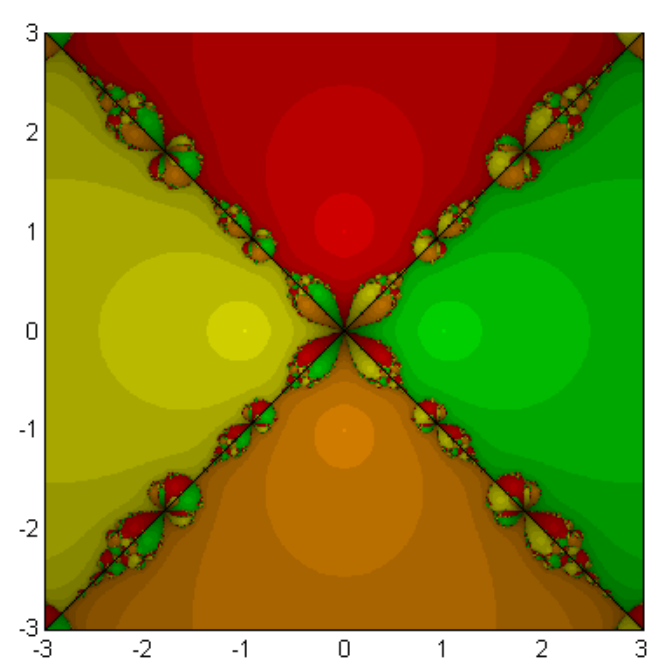

Fig. 35 Our method with $c=1.82$ and $e=0.91$ for the roots of the polynomial $z^{4}-1$.

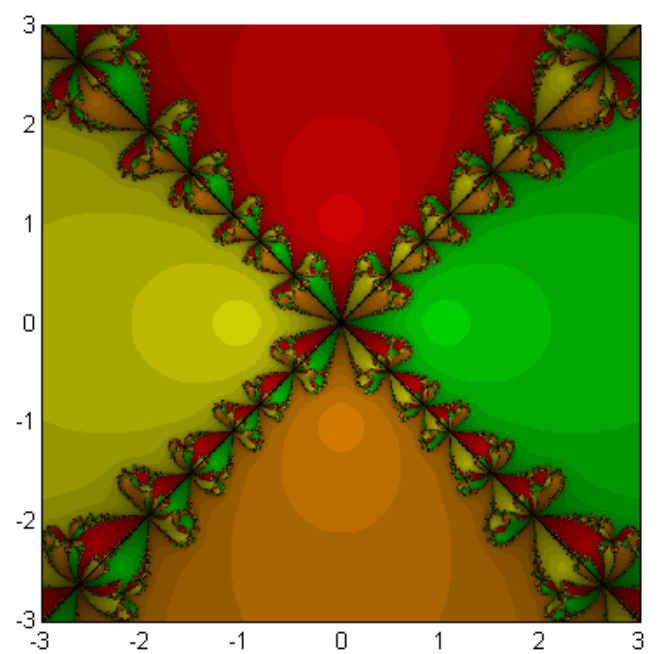

Fig. 36 Jarratt's method with $c=2 / 9$ and $e=2 / 9$ for the roots of the polynomial $z^{4}-1$. 
in one example. We also remove the one with $c=$ $0.5, e=0$, since it was the worst in the second two examples. Lastly, we remove the scheme with $c=0.76, e=0.01$, since it ranked fifth in two examples and mediocre on the third example.

Example 4. In the next example we have taken a polynomial of degree 5 with the 5 roots of unity, i.e.

$$
p_{4}(z)=z^{5}-1 \text {. }
$$

The results are presented in Figs. 37-44. Now the worst is the method with $c=0.75, e=0.25$ (Fig. 40). The scheme with $c=0.25, e=0$ shows large black regions along the basin boundaries (Fig. 38). The best are the three schemes with $c=2 e$ (Figs. 37, 41 and 43). The method with

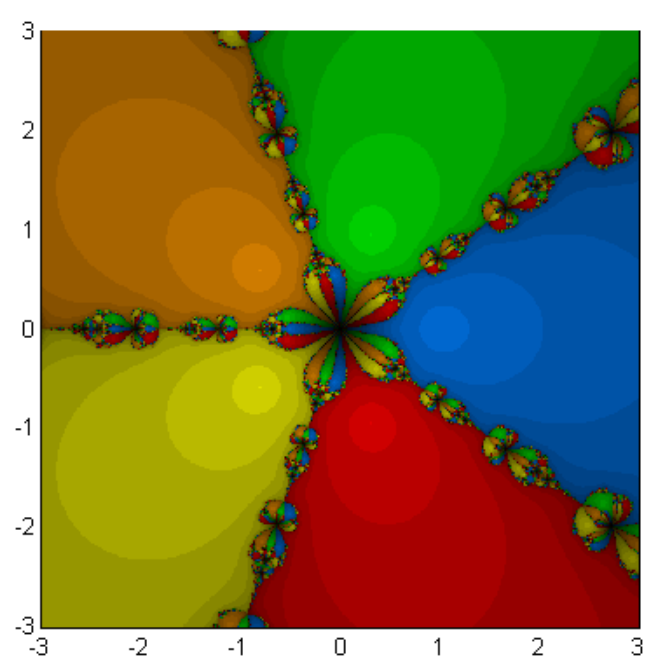

Fig. 37 Our method with $c=0$ and $e=0$ for the roots of the polynomial $z^{5}-1$.

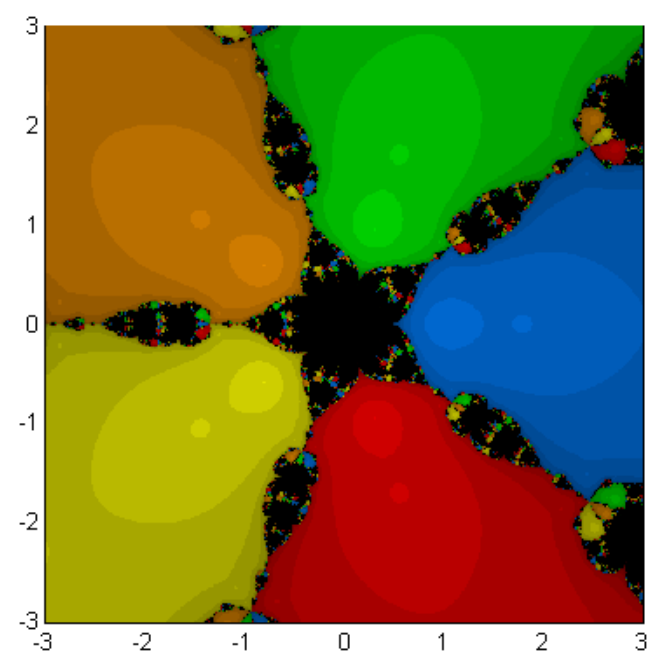

Fig. 38 Our method with $c=0.25$ and $e=0$ for the roots of the polynomial $z^{5}-1$.

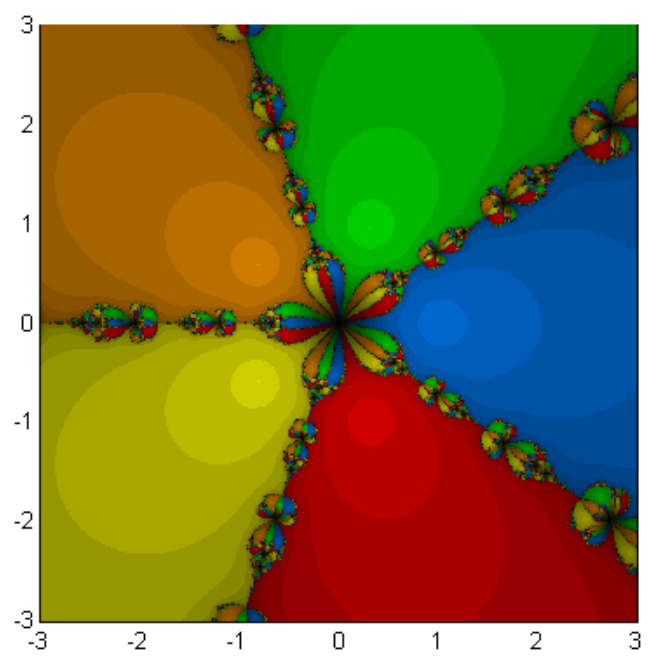

Fig. 39 Jarratt's method with $c=2 / 3$ and $e=1 / 3$ for the roots of the polynomial $z^{5}-1$.

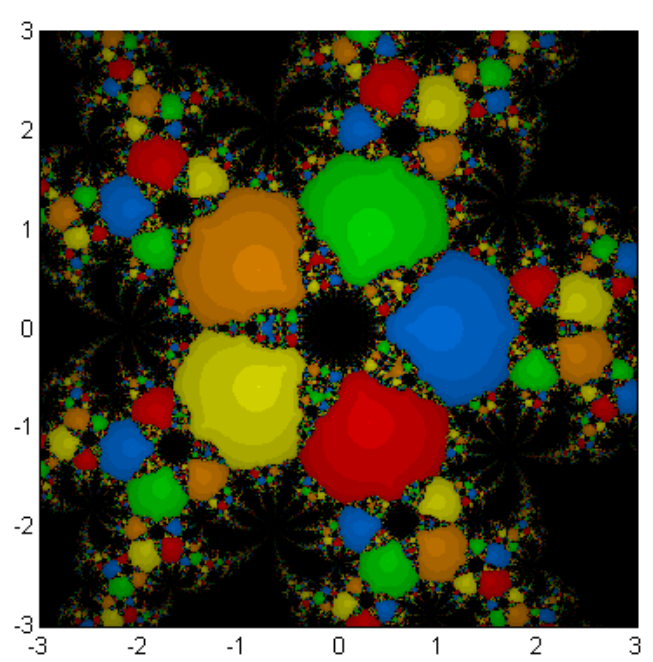

Fig. 40 Our method with $c=0.75$ and $e=0.25$ for the roots of the polynomial $z^{5}-1$.

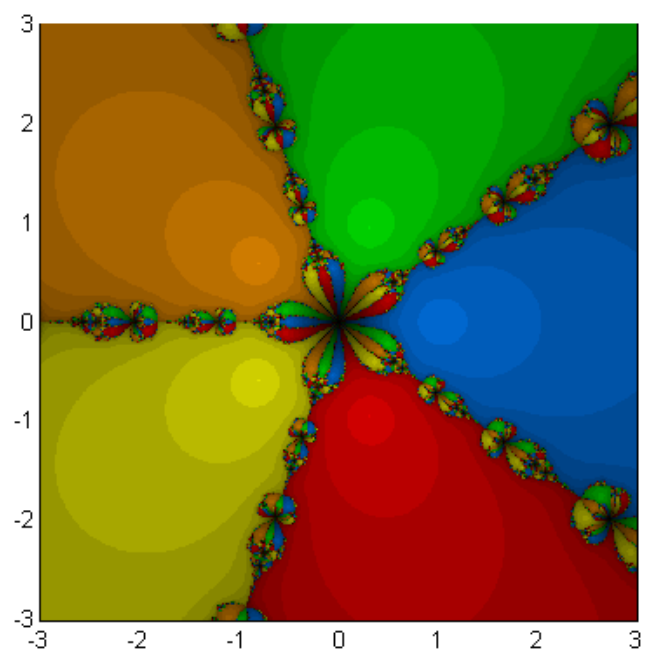

Fig. 41 Our method with $c=0.76$ and $e=0.38$ for the roots of the polynomial $z^{5}-1$. 


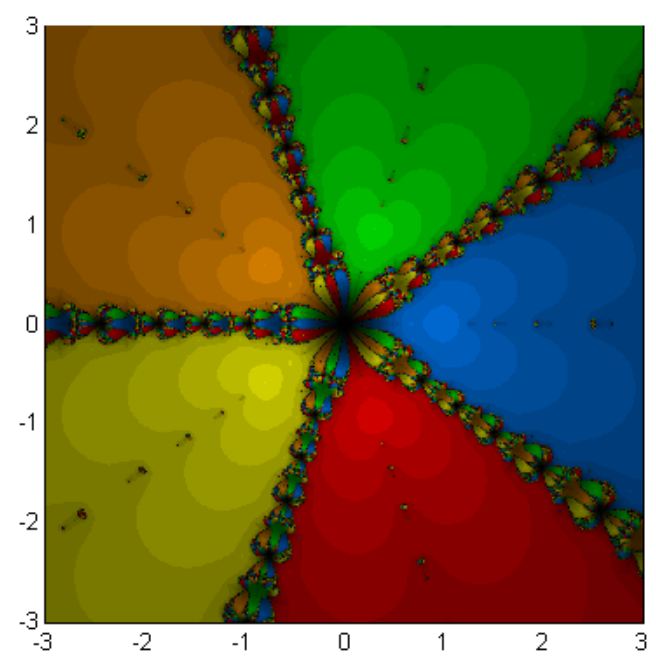

Fig. 42 Our method with $c=1.26$ and $e=0.41$ for the roots of the polynomial $z^{5}-1$.

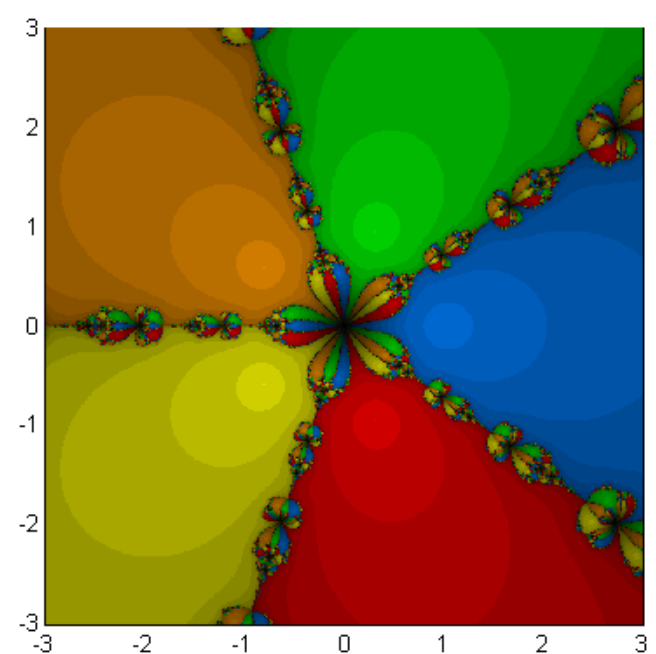

Fig. 43 Our method with $c=1.82$ and $e=0.91$ for the roots of the polynomial $z^{5}-1$.

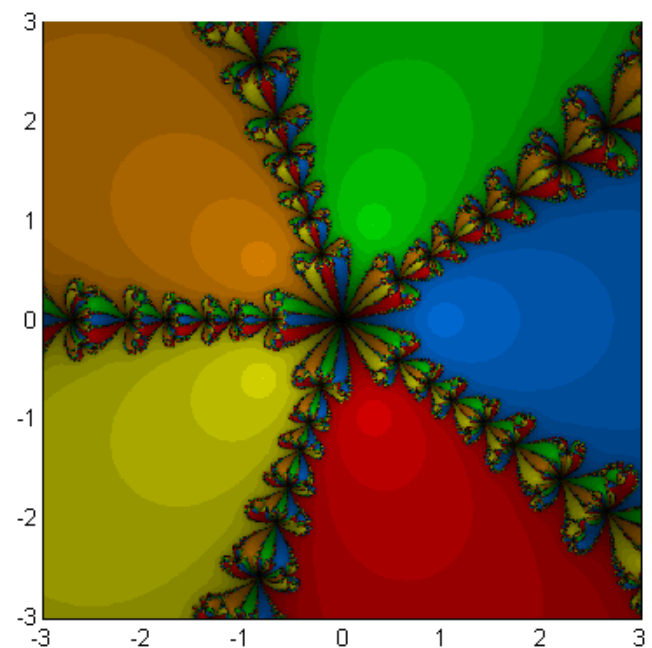

Fig. 44 Jarratt's method with $c=2 / 9$ and $e=2 / 9$ for the roots of the polynomial $z^{5}-1$. $c=1.26, e=0.41$ has black dots inside the basins (Fig. 42).

Example 5. In the next example we took

$$
p_{5}(z)=z^{6}-1 \text {. }
$$

The results are presented in Figs. 45-52. Now the worst is the method with $c=0.75, e=0.25$ (Fig. 48). The scheme with $c=0.25, e=0$ (Fig. 46) shows large black regions along the basin boundaries. The best are the three schemes with $c=2 e$ (Figs. 47, 49 and 51) followed by the one with $c=e=0$ (Fig. 45). The methods with $c=1.26, e=$ 0.41 and $c=2 / 9, e=2 / 9$ have black dots inside the basins and along the basin boundaries (Figs. 50 and $52)$.

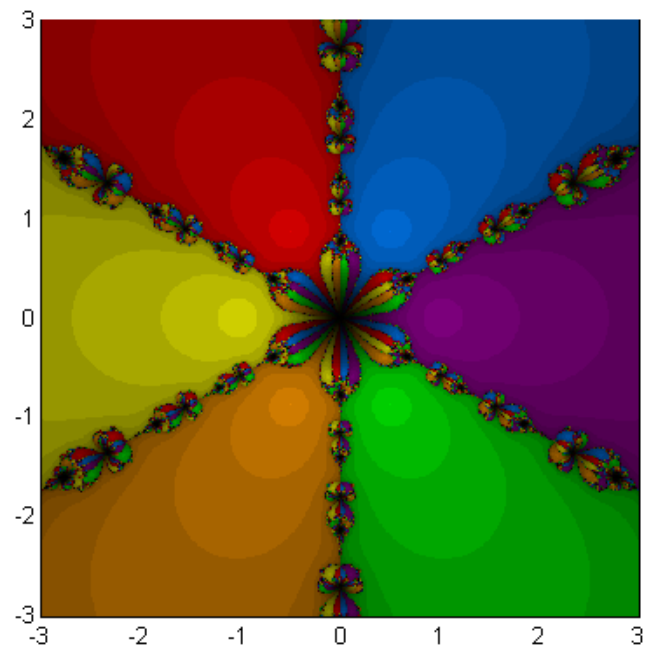

Fig. 45 Our method with $c=0$ and $e=0$ for the roots of the polynomial $z^{6}-1$.

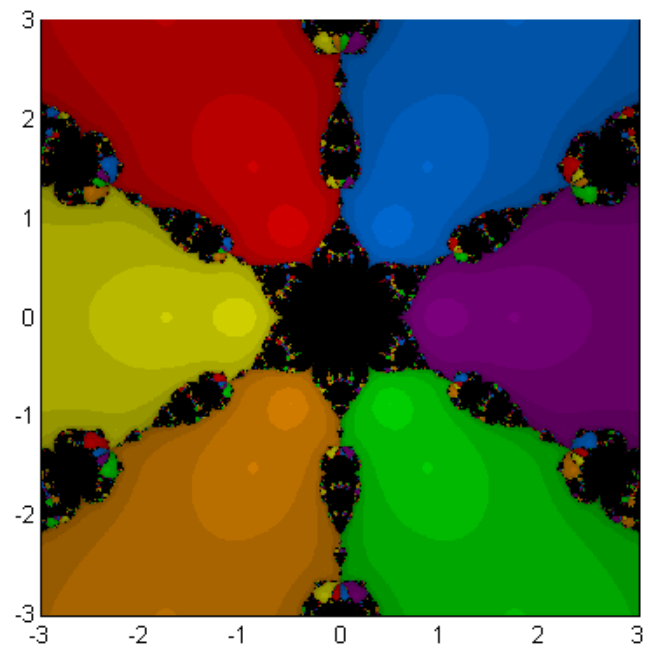

Fig. 46 Our method with $c=0.25$ and $e=0$ for the roots of the polynomial $z^{6}-1$. 


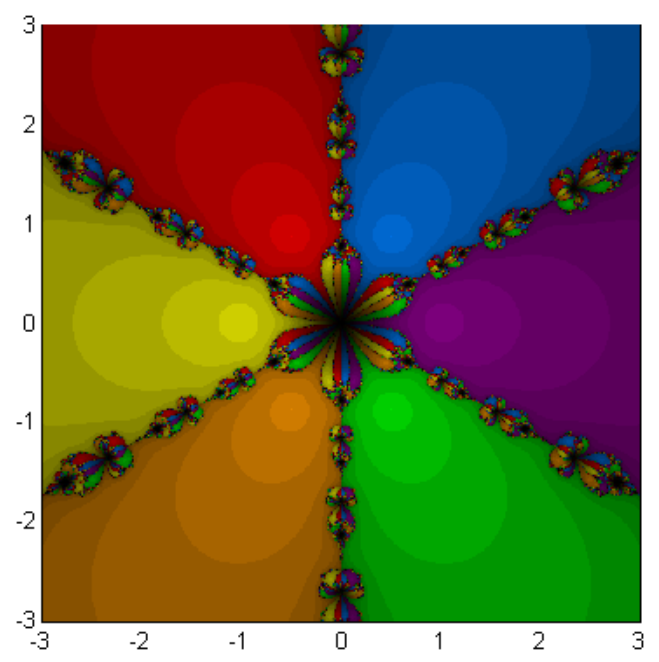

Fig. 47 Jarratt's method with $c=2 / 3$ and $e=1 / 3$ for the roots of the polynomial $z^{6}-1$.

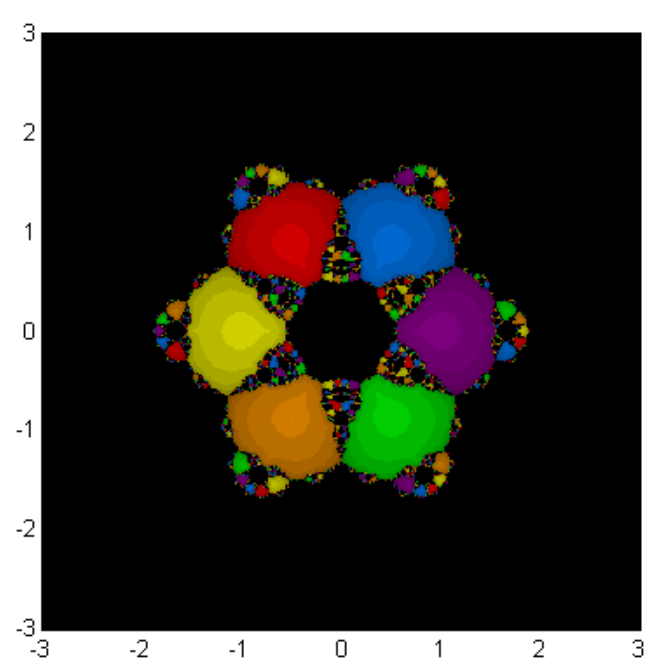

Fig. 48 Our method with $c=0.75$ and $e=0.25$ for the roots of the polynomial $z^{6}-1$.

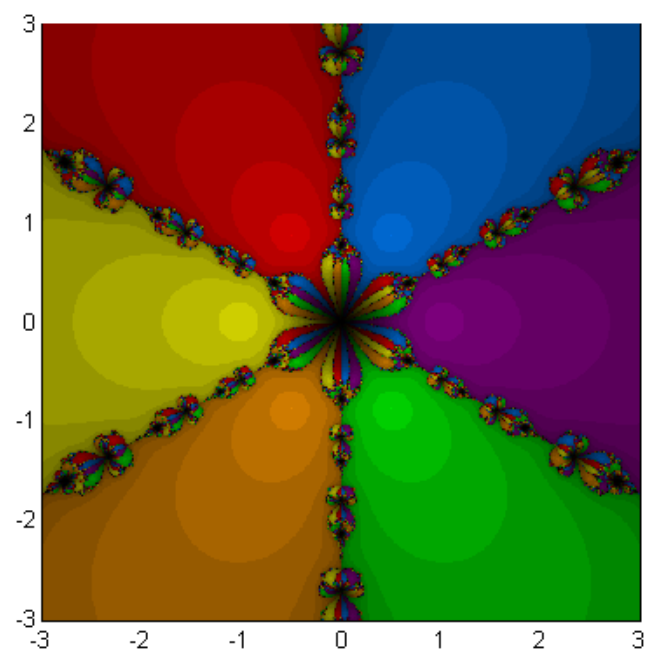

Fig. 49 Our method with $c=0.76$ and $e=0.38$ for the roots of the polynomial $z^{6}-1$.

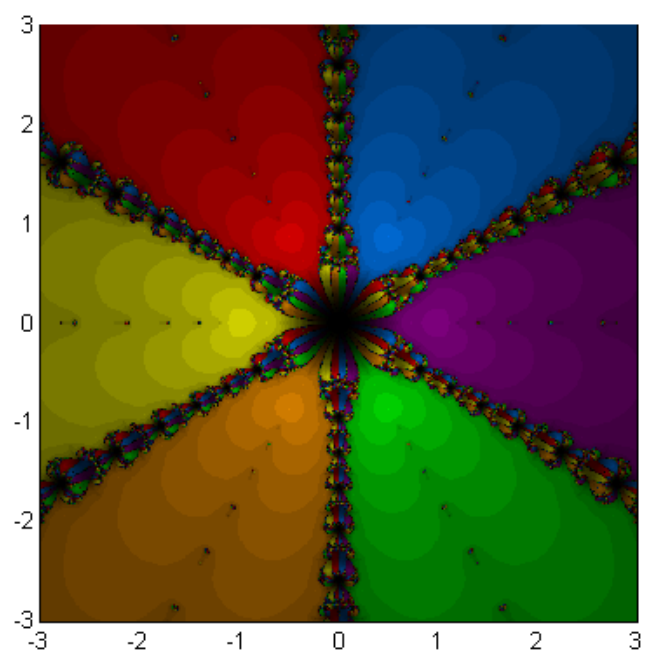

Fig. 50 Our method with $c=1.26$ and $e=0.41$ for the roots of the polynomial $z^{6}-1$.

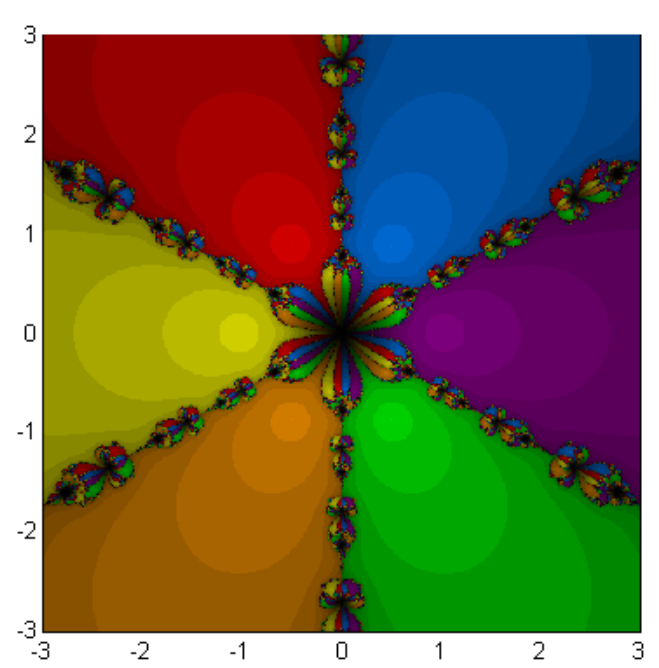

Fig. 51 Our method with $c=1.82$ and $e=0.91$ for the roots of the polynomial $z^{6}-1$.

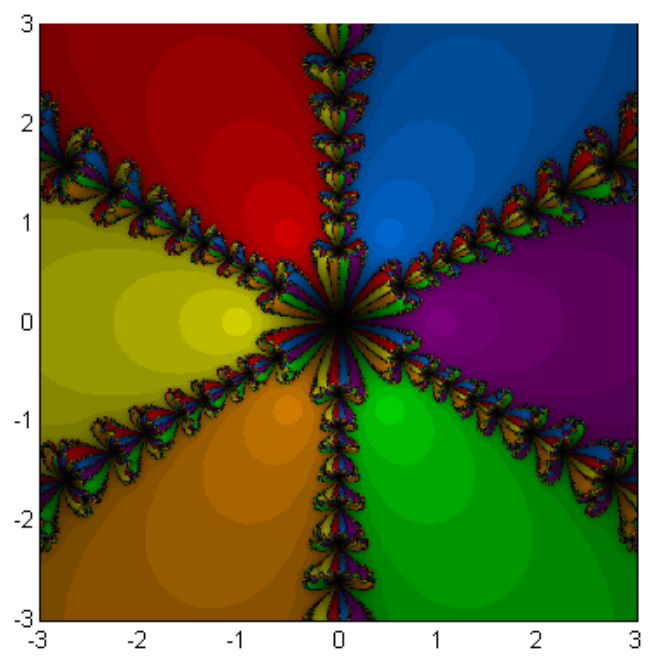

Fig. 52 Jarratt's method with $c=2 / 9$ and $e=2 / 9$ for the roots of the polynomial $z^{6}-1$. 
Example 6. In the last example we took a polynomial of degree 7 having the 7 roots of unity, i.e.

$$
p_{6}(z)=z^{7}-1 \text {. }
$$

The results are presented in Figs. 53-60. The conclusions are the same as in Example 4. Now the worst is the method with $c=0.75, e=0.25$ (Fig. 56). The scheme with $c=0.25, e=0$ shows large black regions along the basin boundaries (Fig. 54). The best are the 3 schemes with $c=2 e$ (Figs. 53, 57 and 59). The methods with $c=1.26, e=0.41$ and $c=2 / 9, e=2 / 9$ have black dots inside the basins (Figs. 58 and 60).

We have tabulated the results in Table 2. We assigned a value between 1 and 6 , where 1 is the best and 6 is the worst. Only the six methods that

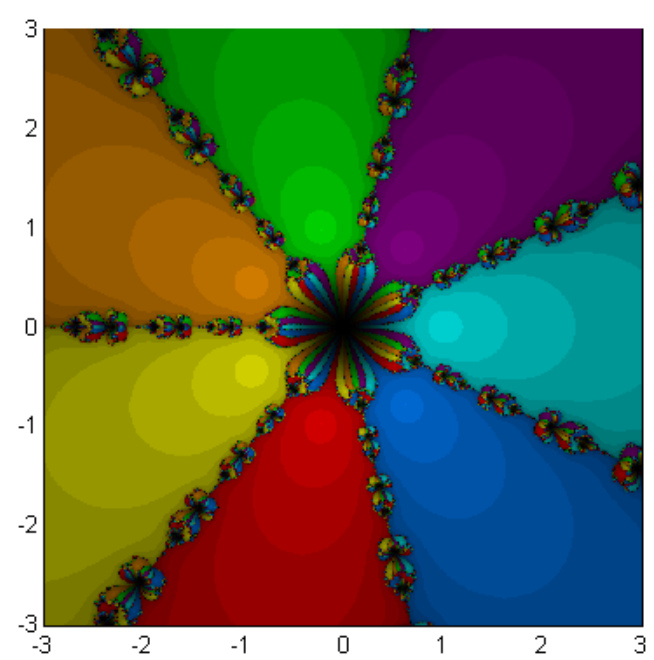

Fig. 53 Our method with $c=0$ and $e=0$ for the roots of the polynomial $z^{7}-1$.

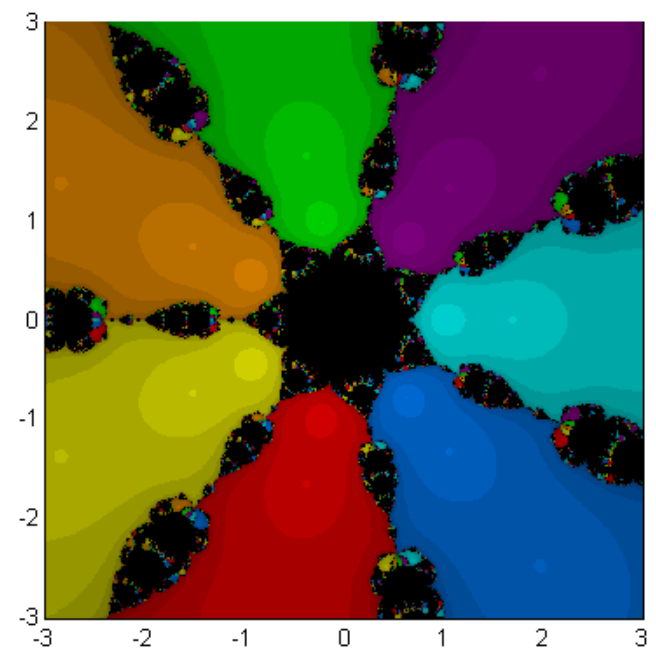

Fig. 54 Our method with $c=0.25$ and $e=0$ for the roots of the polynomial $z^{7}-1$.

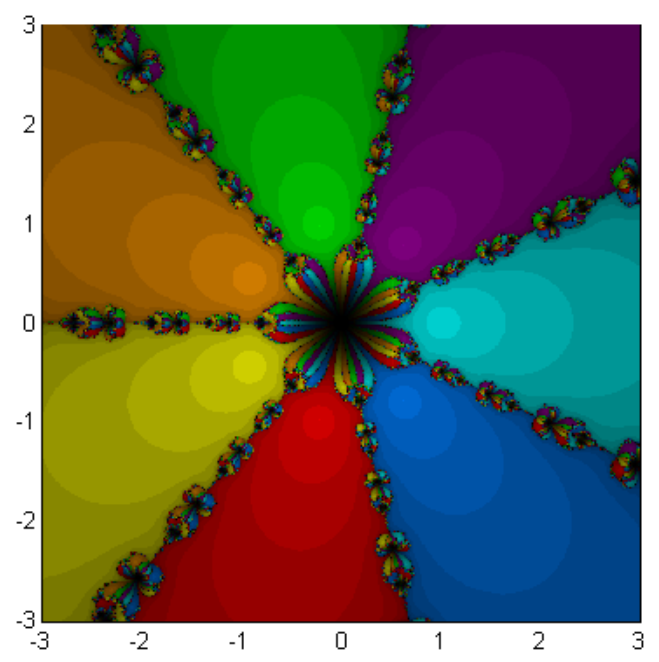

Fig. 55 Jarratt's method with $c=2 / 3$ and $e=1 / 3$ for the roots of the polynomial $z^{6}-1$.

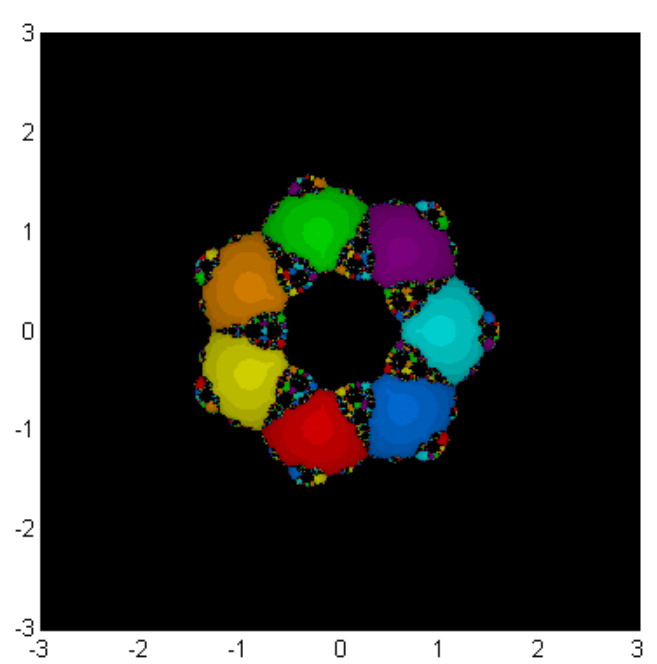

Fig. 56 Our method with $c=0.75$ and $e=0.25$ for the roots of the polynomial $z^{7}-1$.

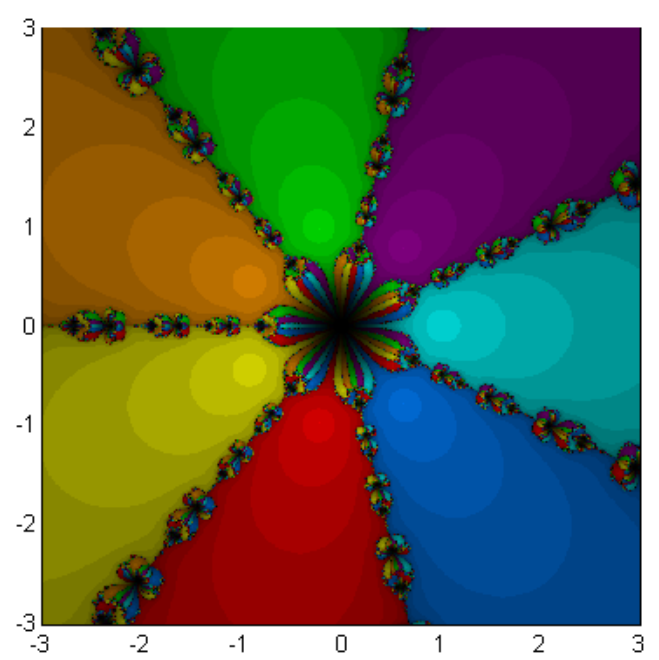

Fig. 57 Our method with $c=0.76$ and $e=0.38$ for the roots of the polynomial $z^{7}-1$. 


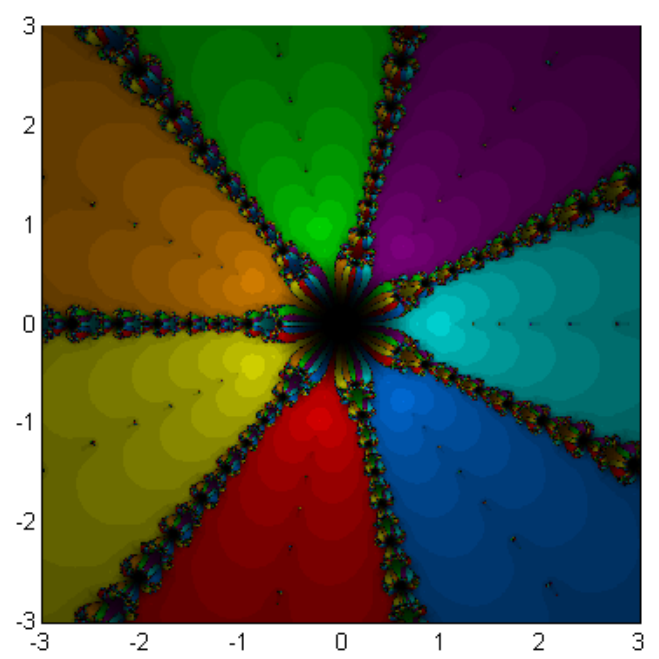

Fig. 58 Our method with $c=1.26$ and $e=0.41$ for the roots of the polynomial $z^{7}-1$.

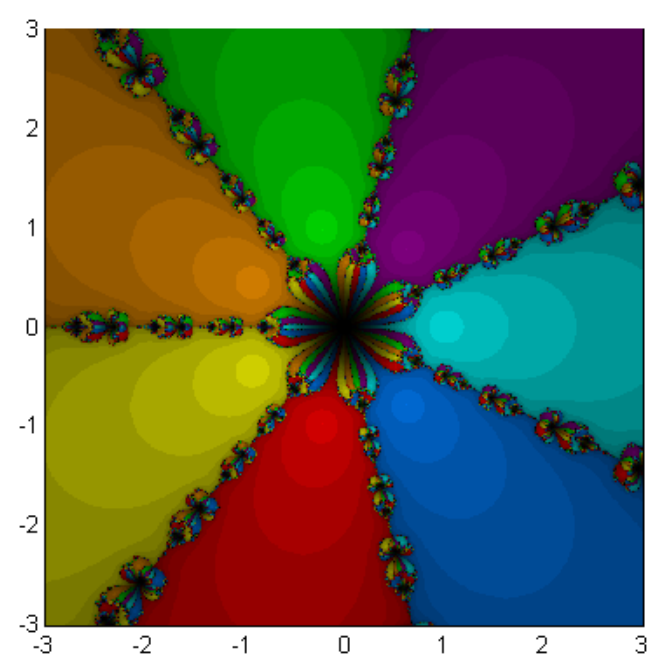

Fig. 59 Our method with $c=1.82$ and $e=0.91$ for the roots of the polynomial $z^{7}-1$.

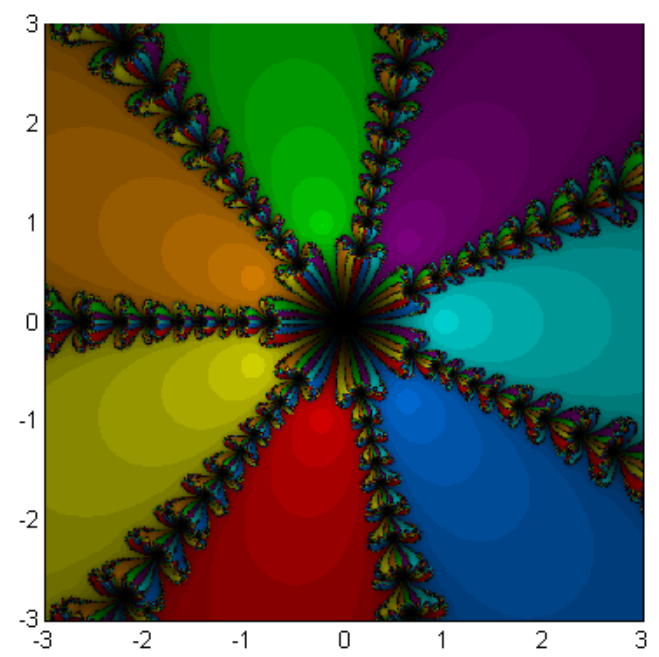

Fig. 60 Jarratt's method with $c=2 / 9$ and $e=2 / 9$ for the roots of the polynomial $z^{7}-1$.
Jarratt's Family of Optimal Fourth-Order Iterative Methods

Table 2 Ordering the Quality of the Basins for Each Example (1-6) and Each Value of $c$ and $e$.

\begin{tabular}{lcccccccc}
\hline \multicolumn{1}{c}{$c$} & $\boldsymbol{e}$ & $\mathbf{E x 1}$ & $\mathbf{E x 2}$ & $\mathbf{E x 3}$ & $\mathbf{E x 4}$ & $\mathbf{E x 5}$ & $\mathbf{E x 6}$ & Total \\
\hline-0.25 & 0 & 6 & 5 & 6 & - & - & - & - \\
0 & 0 & 1 & 1 & 1 & 1 & 2 & 1 & 7 \\
0.25 & 0 & 2 & 4 & 5 & 5 & 5 & 5 & 26 \\
0.5 & 0 & 1 & 6 & 6 & - & - & - & - \\
$2 / 3$ & $1 / 3$ & 1 & 1 & 1 & 1 & 2 & 1 & 7 \\
0.75 & 0.25 & 1 & 2 & 4 & 6 & 6 & 6 & 25 \\
0.76 & 0.01 & 5 & 5 & 3 & - & - & - & - \\
0.76 & 0.38 & 1 & 1 & 1 & 1 & 1 & 1 & 6 \\
1.25 & 0.5 & 3 & 6 & 6 & - & - & - & - \\
1.26 & 0.41 & 4 & 3 & 2 & 4 & 4 & 4 & 21 \\
1.82 & 0.91 & 1 & 1 & 1 & 1 & 1 & 1 & 6 \\
$2 / 9$ & $2 / 9$ & 3 & 2 & 2 & 3 & 3 & 2 & 15 \\
\hline
\end{tabular}

we have ran through all examples will have a total number. The smallest the total the better the overall performance. Based on the results in the table we can conclude that the best methods are those with $c=e=0, c=2 / 3, e=1 / 3, c=0.76, e=0.38$ and $c=1.82, e=0.91$. The other methods are far behind. All four satisfy $c=2 e$ and $S(u)=u^{4}$. One of these is Jarratt's method with $\theta=3 / 2$. The worst is the one with $c=0.75, e=0.25$.

Since these results are subjective, we have decided to use another measure of quality. We have computed the average number of iterations per point. We have taken 360,000 points in the 6 by 6 square centered at the origin. In Table 3 we have listed the average number of iterations. Clearly this number is bounded by 40 , which is the maximum number of iterations allowed. The black points are those requiring that number of iterations and therefore any case having black points will have a high

Table 3 Average Number of Iterations Per Point for Each Example (1-6) and Each Value of $c$ and $e$.

\begin{tabular}{lcccccccc}
\hline \multicolumn{1}{c}{$c$} & $\boldsymbol{e}$ & $\mathbf{E x 1}$ & $\mathbf{E x 2}$ & $\mathbf{E x 3}$ & $\mathbf{E x 4}$ & $\mathbf{E x 5}$ & $\mathbf{E x 6}$ & Total \\
\hline-0.25 & 0 & 4.4 & 7.3 & 10.0 & - & - & - & - \\
0 & 0 & 3.2 & 3.7 & 4.6 & 4.8 & 5.5 & 6.0 & 27.8 \\
0.25 & 0 & 2.8 & 4.8 & 6.4 & 7.4 & 8.9 & 10.0 & 40.3 \\
0.5 & 0 & 3.2 & 23.7 & 31.6 & - & - & - & - \\
$2 / 3$ & $1 / 3$ & 3.2 & 3.7 & 4.6 & 4.8 & 5.5 & 6.0 & 28.8 \\
0.75 & 0.25 & 2.6 & 3.4 & 5.1 & 20.8 & 33.6 & 35.3 & 100.8 \\
0.76 & 0.01 & 4.0 & 6.7 & 7.3 & - & - & - & - \\
0.76 & 0.38 & 3.2 & 3.7 & 5.6 & 4.8 & 5.5 & 6.0 & 28.8 \\
1.25 & 0.5 & 2.8 & 13.6 & 10.8 & - & - & - & - \\
1.26 & 0.41 & 3.5 & 4.9 & 6.1 & 6.8 & 7.9 & 8.9 & 38.1 \\
1.82 & 0.91 & 3.2 & 3.7 & 4.6 & 4.8 & 5.5 & 6.0 & 27.8 \\
$2 / 9$ & $2 / 9$ & 3.5 & 4.5 & 6.0 & 6.4 & 7.4 & 8.5 & 36.3 \\
\hline
\end{tabular}


average. For the eight cases we ran on all six examples we totaled this average number. It is clear that the worst case is that with $c=0.75, e=0.25$. This has a monomial map $S(u)=u^{6}$ with the degree higher than the order of the method. Therefore we conclude that having a monomial map with degree higher than the order is not a good idea. The two cases with a map $S(u)= \pm u^{5}$ did not fare any better. In fact, we have abandoned one after three examples. The one that did best are those with a map $S(u)=u^{4}$ and not $-u^{4}$. Choosing a method with extraneous fixed point farthest from the origin $(c=0.76, e=0.01$ and $c=1.26, e=0.41)$ did not give the best results.

\section{CONCLUSION}

In this paper we have generalized a family of Jarratt's methods and analyzed it. We have shown that it is not enought to choose the extraneous fixed points on the imaginary axis, but we also have to have a conjugacy map which is a monomial of degree $p$, the order of the method.

\section{ACKNOWLEDGMENTS}

This research was supported by Basic Science Research Program through the National Research Foundation of Korea (NRF) funded by the Ministry of Education (NRF-2013R1A1A2005012).

\section{REFERENCES}

1. A. M. Ostrowski, Solution of Equations in Euclidean and Banach Space (Academic Press, New York, 1973).

2. J. F. Traub, Iterative Methods for the Solution of Equations (Chelsea Publishing Company, New York, 1977).

3. B. Neta, Numerical Methods for the Solution of Equations (Net-A-Sof, California, 1983).

4. M. S. Petković, B. Neta, L. D. Petković and J. Džunić, Multipoint Methods for Solving Nonlinear Equations (Elsevier, Waltham, MA, 2013).
5. E. Halley, A new, exact and easy method of finding the roots of equations generally and that without any previous reduction, Philos. Trans. Roy. Soc. Lond. 18 (1694) 136-148.

6. C. Chun, M. Y. Lee, B. Neta and J. Dŭunić, On optimal fourth-order iterative methods free from second derivative and their dynamics, Appl. Math. Comput. 218 (2012) 6427-6438.

7. P. Jarratt, Some fourth order multipoint methods for solving equations, Math. Comp. 20 (1966) 434437 .

8. B. D. Stewart, Attractor basins of various rootfinding methods, M.S. thesis, Naval Postgraduate School, Department of Applied Mathematics, Monterey, CA, June 2001.

9. S. Amat, S. Busquier and S. Plaza, Iterative rootfinding methods, unpublished report, 2004.

10. S. Amat, S. Busquier and S. Plaza, Review of some iterative root-finding methods from a dynamical point of view, Scientia 10 (2004) 3-35.

11. S. Amat, S. Busquier and S. Plaza, Dynamics of a family of third-order iterative methods that do not require using second derivatives, Appl. Math. Comput. 154 (2004) 735-746.

12. S. Amat, S. Busquier and S. Plaza, Dynamics of the King and Jarratt iterations, Aeq. Math. 69 (2005) 212-2236.

13. M. Scott, B. Neta and C. Chun, Basin attractors for various methods, Appl. Math. Comput. 218 (2011) 2584-2599.

14. F. Chircharro, A. Cordero, J. M. Gutiérrez and J. R. Torregrosa, Complex dynamics of derivativefree methods for nonlinear equations, Appl. Math. Comput. 219 (2013) 7023-7035.

15. A. Cordero, J. García-Maimó, J. R. Torregrosa, M. P. Vassileva and P. Vindel, Chaos in King's iterative family, Appl. Math. Lett. 26 (2013) 842-848.

16. B. Neta, M. Scott and C. Chun, Basin of attractions for several methods to find simple roots of nonlinear equations, Appl. Math. Comput. 218 (2012) 1054810556.

17. B. Neta, M. Scott and C. Chun, Basin attractors for various methods for multiple roots, Appl. Math. Comput. 218 (2012) 5043-5066.

18. B. Neta and C. Chun, On a family of Laguerre methods to find multiple roots of nonlinear equations, Appl. Math. Comput. 219 (2013) 10987-11004. 